TRANSACTIONS OF THE

AMERICAN MATHEMATICAL SOCIETY

Volume 361, Number 9, September 2009, Pages 4491-4517

S 0002-9947(09)04765-5

Article electronically published on April 16, 2009

\title{
EXISTENCE AND LIPSCHITZ REGULARITY OF SOLUTIONS TO BOLZA PROBLEMS IN OPTIMAL CONTROL
}

\author{
P. CANNARSA, H. FRANKOWSKA, AND E. M. MARCHINI
}

\begin{abstract}
In this paper we investigate the existence and Lipschitz continuity of optimal trajectories for the autonomous Bolza problem in control theory. The main feature of our results is that they relax the usual fast growth condition for the Lagrangian. Furthermore, we show that optimal solutions do satisfy the maximum principle.
\end{abstract}

\section{INTRODUCTION}

The so-called Bolza problem is such a classical topic in control theory that one might believe nothing interesting can be added to our current knowledge of its basic aspects: existence of solutions, optimality conditions, and regularity of optimal trajectories. In fact, in this paper we will show how combining ideas and techniques that are commonly used to address the above issues, one can extend the comprehension of this problem in a significant way.

To fix ideas, let us seek to minimize the functional

$$
J(x, u)=\int_{0}^{T} L(x(t), u(t)) d t+\ell(x(T)),
$$

over all trajectory/control pairs $(x, u)$ subject to the state equation

$$
\begin{cases}x^{\prime}(t)=f(x(t), u(t)) & \text { for a.e. } t \in I, \\ u(t) \in U & \text { for a.e. } t \in I, \\ x(0) \in C_{0} . & \end{cases}
$$

Here, $I=[0, T]$, while $C_{0} \subset \mathbb{R}^{N}$ and $U \subset \mathbb{R}^{m}$ are closed sets. Moreover, $L$ : $\mathbb{R}^{N} \times U \rightarrow \mathbb{R}$ and $\ell: \mathbb{R}^{N} \rightarrow \mathbb{R}$ are nonnegative functions, $f: \mathbb{R}^{N} \times U \rightarrow \mathbb{R}^{N}$, and $u: I \rightarrow \mathbb{R}^{m}$ is measurable.

The existence of solutions to the above problem is usually obtained under very mild regularity conditions on the data, provided the Lagrangian $L$ is convex with

Received by the editors January 27, 2006.

2000 Mathematics Subject Classification. Primary 49J15, 49J30, 49K15, 49 K30.

Key words and phrases. Optimal control, Bolza problem, existence of minimizers, Lipschitz optimal trajectory, Lipschitz costate.

This work was supported in part by European Community's Human Potential Programme under contract HPRN-CT-2002-00281, Evolution Equations. This research was completed in part while the first and third authors visited the CREA, École Polytechnique, Paris and also while the second author visited the Dipartimento di Matematica, Università di Roma "Tor Vergata".

The third author acknowledges the financial support provided through the European Community's Human Potential Programme under contract HPRN-CT-2002-00281, Evolution Equations.

(C)2009 American Mathematical Society Reverts to public domain 28 years from publication 
respect to $u$, and has superlinear growth at $\infty$, i.e., $L(x, u) \geq \Phi(|u|)$, for some function $\Phi: \mathbb{R} \rightarrow \mathbb{R}$ satisfying

$$
\lim _{r \rightarrow+\infty} \frac{\Phi(r)}{r}=+\infty
$$

In such a framework, the superlinearity of $L$ can be used to derive Lipschitz regularity results for optimal trajectories; see 7, 10. Moreover, the above growth condition can be replaced by a similar growth assumption with respect to $f(x, u)$ still ensuring the existence and Lipschitz continuity of solutions; see [9, 11.

On the other hand, some of the main examples in control theory exhibit functionals with linear growth (such as the brachistocrone problem or the area functional for minimal surfaces of revolution), or even with no growth at all. For such functionals no general existence theory is available, to our knowledge. In fact, several counterexamples to the existence of solutions are known in the literature.

An interesting approach to the existence of solutions for Bolza problems in the calculus of variations, where the final cost $\ell$ is replaced by an end-point constraint, is the one proposed by Clarke in 5. In his paper, which also allows for state constraints, existence is obtained assuming that, for some $k>0$,

$$
\operatorname{ess} \inf _{t \in[0, T]}\left|x^{\prime}(t)\right|<k,
$$

for any admissible arc contained in some level set of the functional, and imposing a separation property on the Hamiltonian $H(x, u, p)=\langle p, u\rangle-L(x, u)$ of the form

$$
\sup _{|x|,|u| \leq k, p \in \partial_{u} L(x, u)} H(x, u, p)<\liminf _{c \rightarrow+\infty} \inf _{|x| \leq k,|u| \geq c, p \in \partial_{u} L(x, u)} H(x, u, p),
$$

where $\partial_{u} L$ denotes the subdifferential of $L$ with respect to $u$. For other ways to relax the superlinear growth condition as well as the convexity of $L$, see also [2, 3] .

In this paper, we will show how to generalize conditions (1.3) and (1.4) to cover Bolza problems of type (1.1)-(1.2) in the absence of state constraints. Like the results of [5], our method applies to some control problems with slow or no growth, as well as superlinear growth of the Lagrangian, ensuring the existence and Lipschitz continuity of optimal trajectories, and - last but not least - the Lipschitz continuity of the corresponding co-states.

Unlike [5], our main results are derived by a simple penalization technique. Indeed, for a fixed $\alpha>1$ and any $\epsilon>0$ we introduce the penalized problem

$$
\min \int_{0}^{T}\left[L(x(t), u(t))+\epsilon|f(x(t), u(t))|^{\alpha}\right] d t+\ell(x(T)),
$$

over all trajectory/control pairs $(x, u)$ of (1.2). Notice that the existence of minimizers $\left(x_{\epsilon}, u_{\epsilon}\right)$ for the above functional is now guaranteed by the results of 9 . Then, an essential step of the proofs consists in getting Lipschitz estimates for $x_{\epsilon}$, uniformly in $\epsilon$. These estimates can be obtained using the fact that $H\left(x_{\epsilon}(\cdot), u_{\epsilon}(\cdot), p_{\epsilon}(\cdot)\right)$ is constant, once we have shown that the norms $\left\|p_{\epsilon}\right\|_{\infty}$ are bounded uniformly in $\epsilon$.

This paper is organized as follows. In Section 2 we explain the setup of the problem, introduce a family of penalized functionals, and give existence and regularity results for 'coercive' optimal control problems. Section 3 contains our main results and their proofs. In Section 4 , we discuss a few examples that illustrate the applicability of this theory. Finally, the Appendix contains the proof of a technical result, obtained in [9] under slightly different assumptions. 
We conclude with the list of notation:

- $W^{1,1}\left(I ; \mathbb{R}^{N}\right)$ denotes the space of absolutely continuous functions from $I$ to $\mathbb{R}^{N}$ and $W^{1, \infty}\left(I ; \mathbb{R}^{N}\right)$ the space of Lipschitz continuous functions from $I$ to $\mathbb{R}^{N}$;

- we define

$$
\mathcal{U}:=\left\{u: I \rightarrow \mathbb{R}^{m} \text { measurable }: u(t) \in U \text {, for a.e. } t \in I\right\} ;
$$

- a pair $(x, u)$ where $x \in W^{1,1}\left(I ; \mathbb{R}^{N}\right)$ and $u \in \mathcal{U}$ is called a trajectory/control pair if $(x, u)$ satisfies (1.2);

- for $a, b \in \mathbb{R}$, we set $a \wedge b:=\min \{a, b\}$;

- given a convex set $U$ and $u \in U, N_{U}(u)$ denotes the normal cone to $U$ at $u$;

- we say that $a: \mathbb{R}^{N} \times \mathbb{R}^{m} \rightarrow \mathbb{R}^{d}$ is locally Lipschitz in $x$ uniformly in $u$ if, for every $R>0$, there exists $C_{R}$ such that, for every $x, y \in B(0, R)$ and every $u \in U$,

$$
|a(x, u)-a(y, u)| \leq C_{R}|x-y|
$$

- given $a: \mathbb{R}^{N} \rightarrow \mathbb{R}, b: \mathbb{R}^{N} \times \mathbb{R}^{m} \rightarrow \mathbb{R}, c: \mathbb{R}^{N} \times \mathbb{R}^{m} \rightarrow \mathbb{R}^{N}$ such that, for every $u \in \mathbb{R}^{m}, a(\cdot), b(\cdot, u)$ and $c(\cdot, u)$ are locally Lipschitz functions, we denote by $\partial a(x), \partial_{x} b(x, u)$ and $\partial_{x} c(x, u)$ respectively their generalized gradients and generalized Jacobian (see [6]) and by $\left(\partial_{x} c\right)^{*}(x, u)$ the adjoint of $\partial_{x} c(x, u)$;

- $\chi_{S}$ denotes the characteristic function of a set $S$;

- for $\alpha>1, \alpha^{\prime}=\frac{\alpha}{\alpha-1}$ denotes the conjugate exponent of $\alpha$;

- $C$ denotes a generic constant that may differ from line to line.

\section{Preliminary Results}

The results of this section are needed for the proof of our main theorems. Let $L, \ell, f$ be given functions such that $L: \mathbb{R}^{N} \times \mathbb{R}^{m} \rightarrow \mathbb{R}$ and $\ell: \mathbb{R}^{N} \rightarrow \mathbb{R}$ are nonnegative, $f: \mathbb{R}^{N} \times \mathbb{R}^{m} \rightarrow \mathbb{R}^{N}, U \subset \mathbb{R}^{m}$ is closed and convex, $C_{0} \subset \mathbb{R}^{N}$ is closed, and, for every $x \in \mathbb{R}^{N}, L(x, \cdot)$ is convex and $f(x, \cdot)$ is differentiable. We set, for $x \in \mathbb{R}^{N}$,

$$
F(x):=\{(f(x, u), L(x, u)+v): u \in U \text { and } v \geq 0\} .
$$

The following assumptions will be in use throughout the paper.

Assumptions ( $\mathbf{H})$ :

i) for some $\alpha>1$ and every $R>0, \exists C_{R}>0$ such that, $\forall x, y \in B(0, R)$ and $\forall u \in U$,

i1) $|\ell(x)-\ell(y)| \leq C_{R}|x-y|$,

i2) $|L(x, u)-L(y, u)| \leq C_{R}|x-y|[1+L(x, u) \wedge L(y, u)]$,

i3) $|f(x, u)-f(y, u)| \leq C_{R}|x-y|\left[1+|f(x, u)| \wedge|f(y, u)|+(L(x, u) \wedge L(y, u))^{1 / \alpha}\right]$;

ii) $\exists \bar{u} \in \mathcal{U}$ such that, for some $\eta \in L^{\alpha}(I ; \mathbb{R})$, for a.e. $t \in I$, and for every $x \in \mathbb{R}^{N}$,

$$
|f(x, \bar{u}(t))| \leq \eta(t)(1+|x|) ;
$$

iii) for every $R>0$, there exists $m_{R} \in L^{1}(I ; \mathbb{R})$ such that, for a.e. $t \in I$,

$$
\sup _{x \in B(0, R)} L(x, \bar{u}(t)) \leq m_{R}(t) ;
$$

iv) for every $x \in \mathbb{R}^{N}, F(x)$ is closed and convex. 
Assumptions $\left(\mathbf{H}^{\prime}\right)$ : the same as assumptions (H) with i3) replaced by

i $\left.3^{\prime}\right)|f(x, u)-f(y, u)| \leq C_{R}|x-y|\left[1+(L(x, u) \wedge L(y, u))^{1 / \alpha}\right]$.

Remark 2.1. Notice that assumptions $\left(\mathrm{H}^{\prime}\right)$ imply assumptions $(\mathrm{H})$. So, every result obtained assuming $(\mathrm{H})$ still holds true under $\left(\mathrm{H}^{\prime}\right)$.

Remark 2.2. From assumptions ii), iii) it follows that the infimum for problem (1.1)-(1.2) is finite.

To show this fact let us fix $x_{0} \in C_{0}$. Owing to the sublinear growth condition ii), we conclude that there exists $\bar{x} \in W^{1, \alpha}\left(I ; \mathbb{R}^{N}\right)$ such that

$$
\left\{\begin{array}{l}
\bar{x}^{\prime}(t)=f(\bar{x}(t), \bar{u}(t)) \quad \text { a.e. } t \in I, \\
\bar{x}(0)=x_{0} .
\end{array}\right.
$$

Let $\bar{R}>0$ be such that $\bar{x}(t) \in B(0, \bar{R}), \forall t \in I$. Since, by iii), $\exists m_{\bar{R}} \in L^{1}(I ; \mathbb{R})$ such that, for a.e. $t \in I, \sup _{x \in B(0, \bar{R})} L(x, \bar{u}(t)) \leq m_{\bar{R}}(t)$, it follows that $J(\bar{x}, \bar{u})<+\infty$.

Proposition 2.3. Assume (H). Then

$$
\begin{aligned}
\inf \{ & \left.J(x, u):(x, u) \text { solves (1.2) and } x^{\prime} \in L^{1}\left(I ; \mathbb{R}^{N}\right)\right\} \\
\quad & \inf \left\{J(x, u):(x, u) \text { solves (1.2) and } x^{\prime} \in L^{\alpha}\left(I ; \mathbb{R}^{N}\right)\right\} .
\end{aligned}
$$

Proof. Let $(\hat{x}, \hat{u})$ be a solution of (1.2) such that $\hat{x}^{\prime} \in L^{1}\left(I ; \mathbb{R}^{N}\right)$, and $J(\hat{x}, \hat{u})<+\infty$. Define, for every $n \in \mathbb{N}$,

$$
u_{n}(t)= \begin{cases}\hat{u}(t) & \text { if }|f(\hat{x}(t), \hat{u}(t))| \leq n, \\ \bar{u}(t) & \text { otherwise }\end{cases}
$$

where $\bar{u}$ is as in $(\mathrm{H})$, and consider the system

$$
\left\{\begin{array}{l}
x^{\prime}(t)=f\left(x(t), u_{n}(t)\right) \quad \text { for a.e. } t \in I \\
x(0)=\hat{x}(0)
\end{array}\right.
$$

We claim that (2.3) has a solution $x_{n}$ such that, for every $n \in \mathbb{N}, x_{n}^{\prime} \in L^{\alpha}\left(I ; \mathbb{R}^{N}\right)$ and, when $n \rightarrow+\infty, x_{n} \rightarrow \hat{x}$ uniformly in $I$. Indeed, set, for $t \in I$,

$$
z_{n}(t)=\hat{x}(0)+\int_{0}^{t} f\left(\hat{x}(s), u_{n}(s)\right) d s .
$$

Then $z_{n} \in W^{1,1}\left(I ; \mathbb{R}^{N}\right), z_{n}^{\prime}(t)=f\left(\hat{x}(t), u_{n}(t)\right)$, for a.e. $t \in I$, and

$$
\begin{aligned}
\left\|\hat{x}-z_{n}\right\|_{\infty} & \leq \int_{0}^{T}\left|f(\hat{x}(s), \hat{u}(s))-f\left(\hat{x}(s), u_{n}(s)\right)\right| d s \\
& =\int_{\{s:|f(\hat{x}(s), \hat{u}(s))|>n\}}|f(\hat{x}(s), \bar{u}(s))-f(\hat{x}(s), \hat{u}(s))| d s .
\end{aligned}
$$

Since $f(\hat{x}(\cdot), \bar{u}(\cdot)), f(\hat{x}(\cdot), \hat{u}(\cdot)) \in L^{1}\left(I ; \mathbb{R}^{N}\right)$, we obtain that $z_{n} \rightarrow \hat{x}$ uniformly in $I$. So, for $n$ sufficiently large, $\left\|\hat{x}-z_{n}\right\|_{\infty} \leq 1$. Define, for a.e. $t \in I$,

$$
\gamma(t)=\left|z_{n}^{\prime}(t)-f\left(z_{n}(t), u_{n}(t)\right)\right| .
$$

Setting $R=\|\hat{x}\|_{\infty}+1$, from (H) i) we deduce that

$$
\gamma(t) \leq C_{R}\left|\hat{x}(t)-z_{n}(t)\right|\left[1+\left|f\left(\hat{x}(t), u_{n}(t)\right)\right|+L\left(\hat{x}(t), u_{n}(t)\right)^{1 / \alpha}\right] .
$$


Since, from the definition of $u_{n}$, there exists $C>0$ such that

$$
\begin{aligned}
& \left\|1+\left|f\left(\hat{x}(\cdot), u_{n}(\cdot)\right)\right|+L\left(\hat{x}(\cdot), u_{n}(\cdot)\right)^{1 / \alpha}\right\|_{L^{1}} \leq T+\|f(\hat{x}(\cdot), \hat{u}(\cdot))\|_{L^{1}} \\
& \quad+\|f(\hat{x}(\cdot), \bar{u}(\cdot))\|_{L^{1}}+\|L(\hat{x}(\cdot), \hat{u}(\cdot))\|_{L^{1}}^{1 / \alpha}+\|L(\hat{x}(\cdot), \bar{u}(\cdot))\|_{L^{1}}^{1 / \alpha} \leq C,
\end{aligned}
$$

for $n$ sufficiently large, we have that

$$
\begin{aligned}
\int_{0}^{T} \gamma(t) e^{\left(\int_{t}^{T} C_{R}\left[1+\left|f\left(\hat{x}(s), u_{n}(s)\right)\right|+L\left(\hat{x}(s), u_{n}(s)\right)^{1 / \alpha}\right] d s\right)} d t \\
\quad \leq \int_{0}^{T} C_{R}\left|\hat{x}(t)-z_{n}(t)\right|\left[1+\left|f\left(\hat{x}(t), u_{n}(t)\right)\right|+L\left(\hat{x}(t), u_{n}(t)\right)^{1 / \alpha}\right] e^{C_{R} C} d t \\
\quad \leq \hat{C}\left\|\hat{x}-z_{n}\right\|_{\infty} \leq 1,
\end{aligned}
$$

for some $\hat{C}>0$. Applying Filippov's Theorem (see, for instance, 12] we deduce that there exists a solution $x_{n}$ to (2.3) such that $\left\|x_{n}-z_{n}\right\|_{\infty} \leq 1$ and, for a.e. $t \in I$,

$$
\begin{aligned}
\left|x_{n}^{\prime}(t)-\hat{x}^{\prime}(t)\right| \leq & \left|x_{n}^{\prime}(t)-z_{n}^{\prime}(t)\right|+\left|z_{n}^{\prime}(t)-\hat{x}^{\prime}(t)\right| \\
= & \left|f\left(x_{n}(t), u_{n}(t)\right)-f\left(\hat{x}(t), u_{n}(t)\right)\right|+\left|f\left(\hat{x}(t), u_{n}(t)\right)-f(\hat{x}(t), \hat{u}(t))\right| \\
\leq & C_{R+1}\left|x_{n}(t)-\hat{x}(t)\right|\left[1+\left|f\left(\hat{x}(t), u_{n}(t)\right)\right|+L\left(\hat{x}(t), u_{n}(t)\right)^{1 / \alpha}\right] \\
& +\left|f\left(\hat{x}(t), u_{n}(t)\right)-f(\hat{x}(t), \hat{u}(t))\right| .
\end{aligned}
$$

Set $R_{n}=\|\hat{x}\|_{\infty}+\left\|x_{n}\right\|_{\infty}$. From (2.2), (H) i) and ii), for a.e. $t \in I$,

$$
\left|f\left(x_{n}(t), u_{n}(t)\right)\right| \leq C_{R_{n}} R_{n}\left[1+n+L(\hat{x}(t), \hat{u}(t))^{1 / \alpha}\right]+\eta(t)\left(1+R_{n}\right) .
$$

So, $x_{n}^{\prime} \in L^{\alpha}\left(I ; \mathbb{R}^{N}\right)$. By (2.4) and Gronwall's Lemma we obtain that

$$
\begin{aligned}
\left\|x_{n}-\hat{x}\right\|_{\infty} & \leq C \int_{0}^{T}\left|f\left(\hat{x}(s), u_{n}(s)\right)-f(\hat{x}(s), \hat{u}(s))\right| d s \\
& =C \int_{\{s:|f(\hat{x}(s), \hat{u}(s))|>n\}}|f(\hat{x}(\sigma), \bar{u}(\sigma))-f(\hat{x}(\sigma), \hat{u}(\sigma))| d \sigma .
\end{aligned}
$$

Therefore $x_{n} \rightarrow \hat{x}$ uniformly in $I$, as $n \rightarrow+\infty$. Let $R>0$ be such that, for every $n \in \mathbb{N}$ and every $t \in I, x_{n}(t), \hat{x}(t) \in B(0, R)$. Then,

$$
L\left(x_{n}(t), u_{n}(t)\right)-L\left(\hat{x}(t), u_{n}(t)\right) \leq C_{R}\left|x_{n}(t)-\hat{x}(t)\right|\left[1+L\left(\hat{x}(t), u_{n}(t)\right)\right] .
$$

Also, for some $C>0$,

$$
\left\|1+L\left(\hat{x}(\cdot), u_{n}(\cdot)\right)\right\|_{L^{1}} \leq T+\|L(\hat{x}(\cdot), \hat{u}(\cdot))\|_{L^{1}}+\|L(\hat{x}(\cdot), \bar{u}(\cdot))\|_{L^{1}} \leq C .
$$

Therefore, owing to $(\mathrm{H}) \mathrm{i}$ ), we have

$$
\begin{aligned}
& \int_{0}^{T}\left[L\left(x_{n}(t), u_{n}(t)\right)-L(\hat{x}(t), \hat{u}(t))\right] d t \\
& =\int_{0}^{T}\left[L\left(x_{n}(t), u_{n}(t)\right)-L\left(\hat{x}(t), u_{n}(t)\right)\right] d t+\int_{0}^{T}\left[L\left(\hat{x}(t), u_{n}(t)\right)-L(\hat{x}(t), \hat{u}(t))\right] d t \\
& \leq C\left\|x_{n}-\hat{x}\right\|_{\infty}+\int_{\{t \in I:|f(\hat{x}(t), \hat{u}(t))|>n\}}[L(\hat{x}(t), \bar{u}(t))-L(\hat{x}(t), \hat{u}(t))] d t,
\end{aligned}
$$

for some $C>0$. Since, for every $n \in \mathbb{N}$,

$$
\inf \left\{J(x, u):(x, u) \text { solves (1.2) and } x^{\prime} \in L^{\alpha}\left(I ; \mathbb{R}^{N}\right)\right\} \leq J\left(x_{n}, u_{n}\right) \text {, }
$$


passing to the limit, we obtain

$$
\inf \left\{J(x, u):(x, u) \text { solves (1.2) and } x^{\prime} \in L^{\alpha}\left(I ; \mathbb{R}^{N}\right)\right\} \leq J(\hat{x}, \hat{u}) .
$$

So, (2.1) holds.

For every $\epsilon>0$, consider the problem

$$
\text { minimize } \quad J_{\epsilon}(x, u)=\int_{0}^{T}\left[L(x(t), u(t))+\frac{\epsilon}{\alpha}|f(x(t), u(t))|^{\alpha}\right] d t+\ell(x(T))
$$

over all trajectory/control pairs $(x, u)$ satisfying (1.2). For $(x, u) \in \mathbb{R}^{N} \times \mathbb{R}^{m}$, set

$$
\left\{\begin{array}{l}
L_{\epsilon}(x, u)=L(x, u)+\frac{\epsilon}{\alpha}|f(x, u)|^{\alpha}, \\
F_{\epsilon}(x)=\left\{\left(f(x, u), L_{\epsilon}(x, u)+v\right): u \in U \text { and } v \geq 0\right\} .
\end{array}\right.
$$

Lemma 2.4. Let $\epsilon>0$ and $x \in \mathbb{R}^{N}$. If (H) iv) is satisfied, then $F_{\epsilon}(x)$ is closed and convex.

Proof. The closedness of $F_{\epsilon}(x)$ follows easily from the closedness of $F(x)$.

We prove that $F_{\epsilon}(x)$ is convex. For $i=1,2$, let

$$
y_{i}=\left(f\left(x, u_{i}\right), L_{\epsilon}\left(x, u_{i}\right)+v_{i}\right) .
$$

We have to show that, for every $\lambda \in(0,1)$, there exist $u_{\lambda} \in U, v_{\lambda} \geq 0$ such that

$$
\lambda y_{1}+(1-\lambda) y_{2}=\left(f\left(x, u_{\lambda}\right), L_{\epsilon}\left(x, u_{\lambda}\right)+v_{\lambda}\right) .
$$

Since $F(x)$ is convex, there exist $\bar{u}_{\lambda} \in U, \bar{v}_{\lambda} \geq 0$ such that

$$
\left\{\begin{array}{l}
\lambda f\left(x, u_{1}\right)+(1-\lambda) f\left(x, u_{2}\right)=f\left(x, \bar{u}_{\lambda}\right) \\
\lambda\left(L\left(x, u_{1}\right)+v_{1}\right)+(1-\lambda)\left(L\left(x, u_{2}\right)+v_{2}\right)=L\left(x, \bar{u}_{\lambda}\right)+\bar{v}_{\lambda} .
\end{array}\right.
$$

Moreover, $\left|f\left(x, \bar{u}_{\lambda}\right)\right|^{\alpha} \leq \lambda\left|f\left(x, u_{1}\right)\right|^{\alpha}+(1-\lambda)\left|f\left(x, u_{2}\right)\right|^{\alpha}$, so that there exists $v \geq 0$ such that

$$
\left|f\left(x, \bar{u}_{\lambda}\right)\right|^{\alpha}+v=\lambda\left|f\left(x, u_{1}\right)\right|^{\alpha}+(1-\lambda)\left|f\left(x, u_{2}\right)\right|^{\alpha} .
$$

Setting $u_{\lambda}=\bar{u}_{\lambda}, v_{\lambda}=\bar{v}_{\lambda}+\frac{\epsilon}{\alpha} v$, the claim follows.

Lemma 2.5. Let $\left\{\left(x_{n}, u_{n}\right)\right\}_{n \in \mathbb{N}}$ be a sequence in $W^{1,1}\left(I ; \mathbb{R}^{N}\right) \times \mathcal{U}$ satisfying (1.2) and

$$
J_{\epsilon}\left(x_{n}, u_{n}\right) \leq c \quad \text { and } \quad\left|x_{n}(0)\right| \leq c, \quad \text { for some } c>0 .
$$

Then, there exist $x \in W^{1,1}\left(I ; \mathbb{R}^{N}\right)$, with $x(0) \in C_{0}$, and a subsequence $\left\{x_{n_{i}}\right\}_{i \in \mathbb{N}}$ such that

$$
\text { as } i \rightarrow \infty, \quad \begin{cases}x_{n_{i}} \rightarrow x & \text { uniformly, } \\ x_{n_{i}}^{\prime} \rightarrow x^{\prime} & \text { weakly in } L^{1} .\end{cases}
$$

Moreover, the sequence $\left\{f\left(x_{n}(\cdot), u_{n}(\cdot)\right)\right\}_{n \in \mathbb{N}}$ is equi-integrable in I.

Since $L_{\epsilon}(x, u) \geq \frac{\epsilon}{\alpha}|f(x, u)|^{\alpha}$, for every $(x, u) \in \mathbb{R}^{N} \times U$, the previous lemma is a consequence of [9, Lemma 4]. In fact, in the above reference, the equi-integrability of $\left\{f\left(x_{n}(\cdot), u_{n}(\cdot)\right)\right\}_{n \in \mathbb{N}}$ is not stated explicitly but follows from the proof. 
Lemma 2.6. Assume (H). Suppose that for some $k>0$ and some trajectory/control pair $\left(x_{1}, u_{1}\right)$ such that

$$
x_{1}^{\prime} \in L^{\alpha}\left(I ; \mathbb{R}^{N}\right) \quad \text { and } \inf \{J(x, u):(x, u) \text { solves (1.2) }\}<J\left(x_{1}, u_{1}\right)<+\infty \text {, }
$$

the following holds true: for any trajectory/control pair $(x, u)$ satisfying $x(0) \in C_{0}$ and $J(x, u)<J\left(x_{1}, u_{1}\right)$,

$$
\|x\|_{\infty} \leq k
$$

Then, for $\epsilon>0$ sufficiently small $\left(\right.$ say $\epsilon \in\left(0, \epsilon_{0}\right)$ ),

a) there exists an optimal solution $\left(x_{\epsilon}, u_{\epsilon}\right)$ to problem (2.5), (1.2) such that $J\left(x_{\epsilon}, u_{\epsilon}\right)<J\left(x_{1}, u_{1}\right)$;

b) there exist $c_{\epsilon} \in \mathbb{R}$ and $p_{\epsilon} \in W^{1,1}\left(I ; \mathbb{R}^{N}\right)$ such that, for a.e. $t \in I$,

$$
\begin{aligned}
c_{\epsilon} & =\left\langle p_{\epsilon}(t), f\left(x_{\epsilon}(t), u_{\epsilon}(t)\right)\right\rangle-L_{\epsilon}\left(x_{\epsilon}(t), u_{\epsilon}(t)\right) \\
& =\max _{u \in U}\left\{\left\langle p_{\epsilon}(t), f\left(x_{\epsilon}(t), u\right)\right\rangle-L_{\epsilon}\left(x_{\epsilon}(t), u\right)\right\} .
\end{aligned}
$$

Moreover, if $\sup _{\epsilon \in\left(0, \epsilon_{0}\right)}\left\|f\left(x_{\epsilon}(\cdot), u_{\epsilon}(\cdot)\right)\right\|_{L^{\alpha}}<+\infty$, then $\sup _{\epsilon \in\left(0, \epsilon_{0}\right)}\left\|p_{\epsilon}\right\|_{\infty}<+\infty$. Furthermore, if for a sequence $\epsilon_{n} \rightarrow 0^{+}, \sup _{n \in \mathbb{N}}\left[\left\|L\left(x_{\epsilon_{n}}(\cdot), u_{\epsilon_{n}}(\cdot)\right)\right\|_{\infty}+\| f\left(x_{\epsilon_{n}}(\cdot)\right.\right.$, $\left.\left.u_{\epsilon_{n}}(\cdot)\right) \|_{\infty}\right]<+\infty$, then $\sup _{n \in \mathbb{N}}\left\|p_{\epsilon_{n}}^{\prime}\right\|_{\infty}<+\infty$.

Lemma 2.7. Under the assumptions of Lemma 2.6 with $(H)$ replaced by $\left(H^{\prime}\right)$, the functions $p_{\epsilon}$ introduced in b) of Lemma 2.6 satisfy, in addition,

$$
\sup _{\epsilon \in\left(0, \epsilon_{0}\right)}\left\|p_{\epsilon}\right\|_{\infty}<+\infty \text {. }
$$

By Remark 2.2 we know that the infimum for problem (2.5), (1.2) is finite.

The proofs of Lemmas 2.6 and 2.7 follow the lines of the proof of Theorem 3 in 9, but, since the assumptions and conclusions of these lemmas are not the same as in the theorem quoted above, for the sake of completeness we provide their proofs in the Appendix.

\section{THE MAIN RESUltS}

For $(x, u, p) \in \mathbb{R}^{N} \times U \times \mathbb{R}^{N}$, set

$$
P(x, u)=\left\{p \in \mathbb{R}^{N}: f_{u}^{\prime}(x, u)^{*} p \in \partial_{u} L(x, u)+N_{U}(u)\right\},
$$

where $\partial_{u} L(x, u)$ denotes the subdifferential of convex analysis of $L(x, \cdot)$ at $u$. Define

$$
H(x, u, p)=\langle p, f(x, u)\rangle-L(x, u) .
$$

The following theorem ensures the existence and Lipschitzianity of solutions for the Bolza problem introduced in Section 11 the Lipschitzianity of the corresponding co-states and the validity of the maximum principle.

Theorem 3.1. Assume (H). Suppose, for some $k>0$ and some trajectory/control pair $\left(x_{1}, u_{1}\right)$ such that $x_{1}^{\prime} \in L^{\alpha}\left(I ; \mathbb{R}^{N}\right)$ and $+\infty>J\left(x_{1}, u_{1}\right)>\inf J(x, u)$ over all the trajectory/control pairs $(x, u)$ satisfying (1.2), the following holds true:

1) for any trajectory/control pair $(x, u)$ satisfying $x(0) \in C_{0}$ and $J(x, u)<$ $J\left(x_{1}, u_{1}\right)$,

$$
\|x\|_{\infty} \leq k \quad \text { and } \quad \operatorname{essinf}_{t \in I}|f(x(t), u(t))|<k ;
$$

2) $\sup _{\substack{|x| \leq k,|f(x, u)| \leq k \\ p \in P(x, u)}} H(x, u, p)<\liminf _{c \rightarrow+\infty} \inf _{\substack{|x| \leq k,|f(x, u)| \geq c \\ p \in P(x, u)}} H(x, u, p)$. 
Then,

a) problem (1.1)-(1.2) has an optimal solution $\left(x^{*}, u^{*}\right)$ such that $x^{*}$ is Lipschitzian and $L\left(x^{*}(\cdot), u^{*}(\cdot)\right) \in L^{\infty}\left(I ; \mathbb{R}^{N}\right)$;

b) there exist $c \in \mathbb{R}$ and $p \in W^{1, \infty}\left(I ; \mathbb{R}^{N}\right)$ such that, for a.e. $t \in I$,

$$
c=\left\langle p(t), f\left(x^{*}(t), u^{*}(t)\right)\right\rangle-L\left(x^{*}(t), u^{*}(t)\right)=\max _{u \in U}\left\{\left\langle p(t), f\left(x^{*}(t), u\right)\right\rangle-L\left(x^{*}(t), u\right)\right\}
$$

and

$$
-p^{\prime}(t) \in\left(\partial_{x} f\right)^{*}\left(x^{*}(t), u^{*}(t)\right) p(t)-\partial_{x} L\left(x^{*}(t), u^{*}(t)\right) .
$$

The next theorem has the same conclusions but a less restrictive assumption 2). Set, for $\nu>0$,

$$
P_{\nu}(x, u)=P(x, u) \cap \overline{B(0, \nu)} .
$$

Theorem 3.2. Assume $\left(H^{\prime}\right)$. Under the hypotheses of Theorem 3.1, with 2) replaced by

$\left.2^{\prime}\right)$ there exists $\nu_{0}>0$ such that, for every $\nu \geq \nu_{0}$,

$$
\sup _{\substack{|x| \leq k,|f(x, u)| \leq k \\ p \in P_{\nu}(x, u)}} H(x, u, p)<\liminf _{c \rightarrow+\infty} \inf _{\substack{|x| \leq k,|f(x, u)| \geq c \\ p \in P_{\nu}(x, u)}} H(x, u, p),
$$

the same conclusions a) and b) of Theorem 3.1 hold true.

Proof of Theorem 3.1. Step 1. For $\epsilon>0$, let us consider the penalized problem introduced in Section 2. By Lemma 2.6, for every $\epsilon>0$ sufficiently small (say $\epsilon \in\left(0, \epsilon_{0}\right)$ ), problem (2.5), (1.2) admits an optimal solution $\left(x_{\epsilon}, u_{\epsilon}\right)$ such that

$$
J\left(x_{\epsilon}, u_{\epsilon}\right)<J\left(x_{1}, u_{1}\right) .
$$

We will show that there exist a sequence $\left\{\epsilon_{n}\right\}_{n \in \mathbb{N}} \subset\left(0, \epsilon_{0}\right)$ and an arc $x^{*} \in$ $W^{1, \infty}\left(I ; \mathbb{R}^{N}\right)$ such that $x^{*}(0) \in C_{0}$ and, as $n \rightarrow+\infty, \epsilon_{n} \downarrow 0$ and

$$
\begin{cases}x_{\epsilon_{n}} \rightarrow x^{*} & \text { uniformly, } \\ f\left(x_{\epsilon_{n}}(\cdot), u_{\epsilon_{n}}(\cdot)\right) \rightarrow x^{* \prime} & \text { weakly-* in } L^{\infty} .\end{cases}
$$

For this aim, we prove that, for some $c(k)>0$ and for a.e. $t \in I$,

$$
\sup _{\epsilon \in\left(0, \epsilon_{0}\right)}\left\|f\left(x_{\epsilon}(\cdot), u_{\epsilon}(\cdot)\right)\right\|_{\infty} \leq c(k) .
$$

From (3.1) and assumption 1),

$$
\left\|x_{\epsilon}\right\|_{\infty} \leq k, \quad \text { for every } \epsilon \in\left(0, \epsilon_{0}\right),
$$

and the set

$$
A_{\epsilon}=\left\{t \in I: \mid f\left(x_{\epsilon}(t), u_{\epsilon}(t) \mid \leq k\right\}\right.
$$

has positive measure. From Lemma 2.6, there exist $c_{\epsilon} \in \mathbb{R}$ and $p_{\epsilon} \in W^{1,1}\left(I ; \mathbb{R}^{N}\right)$ such that, for a.e. $t \in I$,

$$
\begin{aligned}
c_{\epsilon} & =\left\langle p_{\epsilon}(t), f\left(x_{\epsilon}(t), u_{\epsilon}(t)\right)\right\rangle-L\left(x_{\epsilon}(t), u_{\epsilon}(t)\right)-\frac{\epsilon}{\alpha}\left|f\left(x_{\epsilon}(t), u_{\epsilon}(t)\right)\right|^{\alpha} \\
& =\max _{u \in U}\left\{\left\langle p_{\epsilon}(t), f\left(x_{\epsilon}(t), u\right)\right\rangle-L\left(x_{\epsilon}(t), u\right)-\frac{\epsilon}{\alpha}\left|f\left(x_{\epsilon}(t), u\right)\right|^{\alpha}\right\} .
\end{aligned}
$$

Now, in view of (3.4) , for a.e. $t \in I$, we have that $f_{u}^{\prime}\left(x_{\epsilon}(t), u_{\epsilon}(t)\right)^{*} p_{\epsilon}(t)$ belongs to the set

$$
\partial_{u} L\left(x_{\epsilon}(t), u_{\epsilon}(t)\right)+\epsilon\left|f\left(x_{\epsilon}(t), u_{\epsilon}(t)\right)\right|^{\alpha-1} f_{u}^{\prime}\left(x_{\epsilon}(t), u_{\epsilon}(t)\right)^{*} \phi_{\varepsilon}(t)+N_{U}\left(u_{\epsilon}(t)\right),
$$


where $\phi_{\varepsilon}(\cdot)$ is a measurable function such that

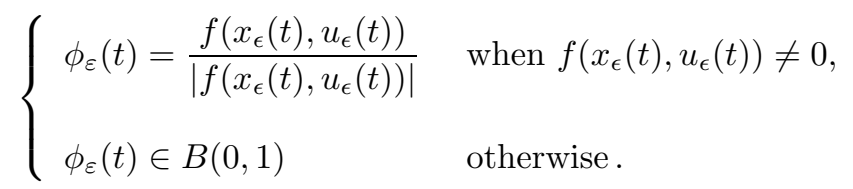

In other words, there exist two measurable functions $q_{\epsilon}, \pi_{\epsilon}: I \rightarrow \mathbb{R}^{m}$ such that, for a.e. $t \in I, q_{\epsilon}(t) \in \partial_{u} L\left(x_{\epsilon}(t), u_{\epsilon}(t)\right), \pi_{\epsilon}(t) \in N_{U}\left(u_{\epsilon}(t)\right)$, and

$$
\begin{aligned}
& f_{u}^{\prime}\left(x_{\epsilon}(t), u_{\epsilon}(t)\right)^{*} p_{\epsilon}(t) \\
& \quad=q_{\epsilon}(t)+\epsilon\left|f\left(x_{\epsilon}(t), u_{\epsilon}(t)\right)\right|^{\alpha-1} f_{u}^{\prime}\left(x_{\epsilon}(t), u_{\epsilon}(t)\right)^{*} \phi_{\varepsilon}(t)+\pi_{\epsilon}(t) .
\end{aligned}
$$

So,

$$
\begin{aligned}
f_{u}^{\prime}\left(x_{\epsilon}(t), u_{\epsilon}(t)\right)^{*} & {\left[p_{\epsilon}(t)-\epsilon\left|f\left(x_{\epsilon}(t), u_{\epsilon}(t)\right)\right|^{\alpha-1} \phi_{\varepsilon}(t)\right] } \\
& \in \partial_{u} L\left(x_{\epsilon}(t), u_{\epsilon}(t)\right)+N_{U}\left(x_{\epsilon}(t)\right),
\end{aligned}
$$

or equivalently,

$$
p_{\epsilon}(t)-\epsilon\left|f\left(x_{\epsilon}(t), u_{\epsilon}(t)\right)\right|^{\alpha-1} \phi_{\varepsilon}(t) \in P\left(x_{\epsilon}(t), u_{\epsilon}(t)\right) .
$$

Applying assumption 2) we deduce that, for some $c(k) \geq k$,

$$
\sup _{\substack{|x| \leq k,|f(x, u)| \leq k \\ p \in P(x, u)}} H(x, u, p)<\inf _{\substack{|x| \leq k,|f(x, u)| \geq c(k) \\ p \in P(x, u)}} H(x, u, p) .
$$

Set

$$
B_{\epsilon}=\left\{t \in I:\left|f\left(x_{\epsilon}(t), u_{\epsilon}(t)\right)\right|>c(k)\right\} .
$$

We will show that $\mu\left(B_{\epsilon}\right)=0$, arguing by contradiction. Indeed, assume that $\mu\left(B_{\epsilon}\right)>0$, and let $a \in A_{\epsilon}$ and $b \in B_{\epsilon}$ be such that (3.4) and (3.5) hold.

Suppose first that $\left|f\left(x_{\epsilon}(a), u_{\epsilon}(a)\right)\right|>0$. By (3.4), we have that

$$
\begin{aligned}
c_{\epsilon} & =\left\langle p_{\epsilon}(a), f\left(x_{\epsilon}(a), u_{\epsilon}(a)\right)\right\rangle-L\left(x_{\epsilon}(a), u_{\epsilon}(a)\right)-\frac{\epsilon}{\alpha}\left|f\left(x_{\epsilon}(a), u_{\epsilon}(a)\right)\right|^{\alpha} \\
& =\left\langle p_{\epsilon}(b), f\left(x_{\epsilon}(b), u_{\epsilon}(b)\right)\right\rangle-L\left(x_{\epsilon}(b), u_{\epsilon}(b)\right)-\frac{\epsilon}{\alpha}\left|f\left(x_{\epsilon}(b), u_{\epsilon}(b)\right)\right|^{\alpha} .
\end{aligned}
$$

Also, by (3.5) and (3.6),

$$
\begin{aligned}
& \left\langle p_{\epsilon}(a)-\epsilon\left|f\left(x_{\epsilon}(a), u_{\epsilon}(a)\right)\right|^{\alpha-2} f\left(x_{\epsilon}(a), u_{\epsilon}(a)\right), f\left(x_{\epsilon}(a), u_{\epsilon}(a)\right)\right\rangle-L\left(x_{\epsilon}(a), u_{\epsilon}(a)\right) \\
& <\left\langle p_{\epsilon}(b)-\epsilon\left|f\left(x_{\epsilon}(b), u_{\epsilon}(b)\right)\right|^{\alpha-2} f\left(x_{\epsilon}(b), u_{\epsilon}(b)\right), f\left(x_{\epsilon}(b), u_{\epsilon}(b)\right)\right\rangle-L\left(x_{\epsilon}(b), u_{\epsilon}(b)\right) .
\end{aligned}
$$

So,

$$
\begin{aligned}
c_{\epsilon}= & \left\langle p_{\epsilon}(a)-\epsilon\left|f\left(x_{\epsilon}(a), u_{\epsilon}(a)\right)\right|^{\alpha-2} f\left(x_{\epsilon}(a), u_{\epsilon}(a)\right), f\left(x_{\epsilon}(a), u_{\epsilon}(a)\right)\right\rangle \\
& -L\left(x_{\epsilon}(a), u_{\epsilon}(a)\right)+\epsilon\left(1-\frac{1}{\alpha}\right)\left|f\left(x_{\epsilon}(a), u_{\epsilon}(a)\right)\right|^{\alpha} \\
< & \left\langle p_{\epsilon}(b)-\epsilon\left|f\left(x_{\epsilon}(b), u_{\epsilon}(b)\right)\right|^{\alpha-2} f\left(x_{\epsilon}(b), u_{\epsilon}(b)\right), f\left(x_{\epsilon}(b), u_{\epsilon}(b)\right)\right\rangle \\
& -L\left(x_{\epsilon}(b), u_{\epsilon}(b)\right)+\epsilon\left(1-\frac{1}{\alpha}\right)\left|f\left(x_{\epsilon}(a), u_{\epsilon}(a)\right)\right|^{\alpha} \\
= & c_{\epsilon}-\epsilon\left(1-\frac{1}{\alpha}\right)\left|f\left(x_{\epsilon}(b), u_{\epsilon}(b)\right)\right|^{\alpha}+\epsilon\left(1-\frac{1}{\alpha}\right)\left|f\left(x_{\epsilon}(a), u_{\epsilon}(a)\right)\right|^{\alpha} .
\end{aligned}
$$

Therefore,

$$
c(k)^{\alpha}<\left|f\left(x_{\epsilon}(b), u_{\epsilon}(b)\right)\right|^{\alpha}<\left|f\left(x_{\epsilon}(a), u_{\epsilon}(a)\right)\right|^{\alpha} \leq k^{\alpha},
$$

in contrast with the choice of $c(k)$. 
Finally, in the case $\left|f\left(x_{\epsilon}(a), u_{\epsilon}(a)\right)\right|=0, p_{\epsilon}(a) \in P\left(x_{\epsilon}(a), u_{\epsilon}(a)\right)$ and, arguing as above, we obtain the contradiction $c(k)^{\alpha}<\left|f\left(x_{\epsilon}(b), u_{\epsilon}(b)\right)\right|^{\alpha}<0$.

So, $\mu\left(B_{\epsilon}\right)=0$ and (3.2) follows. Hence, there exists a sequence $\left\{\epsilon_{n}\right\}_{n \in \mathbb{N}} \subset\left(0, \epsilon_{0}\right)$ and an $\operatorname{arc} x^{*} \in W^{1, \infty}\left(I ; \mathbb{R}^{N}\right)$ such that $x^{*}(0) \in C_{0}$ and, as $n \rightarrow+\infty, \epsilon_{n} \downarrow 0$ and

$$
\begin{cases}x_{\epsilon_{n}} \rightarrow x^{*} & \text { uniformly } \\ f\left(x_{\epsilon_{n}}(\cdot), u_{\epsilon_{n}}(\cdot)\right) \rightarrow x^{* \prime} & \text { weakly-* in } L^{\infty} .\end{cases}
$$

Step 2. We claim that there exists $\xi \in L^{\infty}\left(I ; \mathbb{R}^{N}\right)$ such that, for a subsequence, we have

$$
L\left(x_{\epsilon_{n_{i}}}(\cdot), u_{\epsilon_{n_{i}}}(\cdot)\right) \rightarrow \xi \quad \text { weakly-* in } L^{\infty} .
$$

Consider the elements $c_{\epsilon}$ and $p_{\epsilon}$ defined in (3.4). From (3.2) and Lemma 2.6.

$$
\sup _{\epsilon \in\left(0, \epsilon_{0}\right)}\left\|p_{\epsilon}\right\|_{\infty} \leq M, \quad \text { for some } M>0 \text {. }
$$

Notice that

$$
c_{\epsilon} \geq-\gamma, \quad \text { for every } \epsilon \in\left(0, \epsilon_{0}\right) \text { and for some } \gamma>0 \text {. }
$$

Indeed, fix any $u_{0} \in U$ and let $t \in A_{\epsilon}$ be such that (3.4) holds true. Then

$$
c_{\epsilon} \geq\left\langle p_{\epsilon}(t), f\left(x_{\epsilon}(t), u_{0}\right)\right\rangle-L\left(x_{\epsilon}(t), u_{0}\right)-\frac{\epsilon}{\alpha}\left|f\left(x_{\epsilon}(t), u_{0}\right)\right|^{\alpha} .
$$

From (3.3) and (3.8), since $f\left(\cdot, u_{0}\right)$ and $L\left(\cdot, u_{0}\right)$ are continuous, (3.9) follows. So,

$$
c_{\epsilon}=\left\langle p_{\epsilon}(t), f\left(x_{\epsilon}(t), u_{\epsilon}(t)\right)\right\rangle-L\left(x_{\epsilon}(t), u_{\epsilon}(t)\right)-\frac{\epsilon}{\alpha}\left|f\left(x_{\epsilon}(t), u_{\epsilon}(t)\right)\right|^{\alpha} \geq-\gamma .
$$

By (3.2), (3.8), and (3.10), for every $n \in \mathbb{N}$,

$$
L\left(x_{\epsilon_{n}}(t), u_{\epsilon_{n}}(t)\right) \leq\left|p_{\epsilon_{n}}(t)\right|\left|f\left(x_{\epsilon_{n}}(t), u_{\epsilon_{n}}(t)\right)\right|+\gamma \leq M c(k)+\gamma .
$$

Hence, (3.7) follows.

Step 3. By steps 1,2 there exist $x^{*} \in W^{1, \infty}\left(I ; \mathbb{R}^{N}\right)$ with $x^{*}(0) \in C_{0}, \xi \in$ $L^{\infty}\left(I ; \mathbb{R}^{N}\right)$, and a sequence $\epsilon_{n} \rightarrow 0^{+}$such that, when $n \rightarrow+\infty$,

$$
\begin{cases}x_{\epsilon_{n}} \rightarrow x^{*} & \text { uniformly, } \\ x_{\epsilon_{n}}^{\prime} \rightarrow x^{* \prime} & \text { weakly-* in } L^{\infty}, \\ L\left(x_{\epsilon_{n}}(\cdot), u_{\epsilon_{n}}(\cdot)\right) \rightarrow \xi & \text { weakly-* in } L^{\infty} .\end{cases}
$$

We want to prove that, for a.e. $t \in I$,

$$
\left(x^{* \prime}(t), \xi(t)\right) \in F\left(x^{*}(t)\right) .
$$

Set, for every $t \in I, z_{n}(t)=\left(f\left(x_{\epsilon_{n}}(t), u_{\epsilon_{n}}(t)\right), L\left(x_{\epsilon_{n}}(t), u_{\epsilon_{n}}(t)\right)\right)$ and $z^{*}(t)=$ $\left(x^{* \prime}(t), \xi(t)\right)$. We know that $z_{n} \rightarrow z^{*}$ weakly-* in $L^{\infty}$. By Mazur's Theorem, we can choose functions

$$
w_{n}(t)=\sum_{i=n}^{\infty} a_{n}^{i} z_{i}(t)
$$

(where, $\forall n \geq 1, a_{n}^{i} \geq 0$ are equal to zero except for finitely many $i$ 's, and $\sum_{i=n}^{\infty} a_{n}^{i}=$ 1 ), such that the sequence $\left\{w_{n}\right\}_{n \in \mathbb{N}}$ converges strongly to $z^{*}$ in $L^{1}$. So, taking a subsequence and keeping the same notation, for a.e. $t \in I, \lim _{n \rightarrow+\infty} w_{n}(t)=z^{*}(t)$.

Fix $\delta>0$. Then, for a.e. $t \in I$, there exists $N_{t}$ such that, for every $n \geq N_{t}$, we have

$$
z_{n}(t) \in F\left(x^{*}(t)\right)+\delta B(0,1)
$$


Indeed, from $(\mathrm{H}) \mathrm{i}$ ), for every $n \in \mathbb{N}$ and a.e. $t \in I$,

$$
\begin{aligned}
& \left|f\left(x_{\epsilon_{n}}(t), u_{\epsilon_{n}}(t)\right)-f\left(x^{*}(t), u_{\epsilon_{n}}(t)\right)\right| \\
& \quad \leq C_{k}\left|x_{\epsilon_{n}}(t)-x^{*}(t)\right|\left[1+\left|f\left(x_{\epsilon_{n}}(t), u_{\epsilon_{n}}(t)\right)\right|+L\left(x_{\epsilon_{n}}(t), u_{\epsilon_{n}}(t)\right)^{1 / \alpha}\right]
\end{aligned}
$$

and

$$
\left|L\left(x_{\epsilon_{n}}(t), u_{\epsilon_{n}}(t)\right)-L\left(x^{*}(t), u_{\epsilon_{n}}(t)\right)\right| \leq C_{k}\left|x_{\epsilon_{n}}(t)-x^{*}(t)\right|\left[1+L\left(x_{\epsilon_{n}}(t), u_{\epsilon_{n}}(t)\right)\right] .
$$

So, for $N_{t}$ sufficiently large, (3.13) follows. From (3.13), we obtain that if $w_{n}(t) \in$ $\overline{F\left(x^{*}(t)\right)+\delta B(0,1)}$, then its limit $z^{*}(t)=\left(x^{*^{\prime}}(t), \xi(t)\right)$ belongs to the same convex sets, for a.e. $t \in I$. Since $\delta>0$ is arbitrary,

$$
\left(x^{* \prime}(t), \xi(t)\right) \in \bigcap_{\delta>0} \overline{\left(F\left(x^{*}(t)\right)+\delta B(0,1)\right)}=\overline{F\left(x^{*}(t)\right)}=F\left(x^{*}(t)\right) .
$$

By a measurable selection lemma (see, for instance, [1]), there exist two measurable functions $u^{*}, v^{*}$ such that, for a.e. $t \in I, u^{*}(t) \in U, v^{*}(t) \geq 0$ and

$$
\left\{\begin{array}{l}
x^{* \prime}(t)=f\left(x^{*}(t), u^{*}(t)\right) \\
\xi(t)=L\left(x^{*}(t), u^{*}(t)\right)+v^{*}(t) .
\end{array}\right.
$$

We next claim that

$$
\liminf _{n \rightarrow+\infty} J\left(x_{\epsilon_{n}}, u_{\epsilon_{n}}\right)=\inf \left\{J(x, u):(x, u) \text { solves (1.2) and } x^{\prime} \in L^{\alpha}\left(I ; \mathbb{R}^{N}\right)\right\} .
$$

Indeed, let $(x, u)$ satisfy (1.2) and be such that $x^{\prime} \in L^{\alpha}(I ; \mathbb{R})$. Then we get

$$
\liminf _{n \rightarrow+\infty} J\left(x_{\epsilon_{n}}, u_{\epsilon_{n}}\right) \leq \liminf _{n \rightarrow+\infty} J_{\epsilon_{n}}\left(x_{\epsilon_{n}}, u_{\epsilon_{n}}\right) \leq \liminf _{n \rightarrow+\infty} J_{\epsilon_{n}}(x, u)=J(x, u) .
$$

By (3.12) and (3.14) we have that

$$
\begin{aligned}
\int_{0}^{T}\left[L\left(x^{*}(t), u^{*}(t)\right)\right. & \left.+v^{*}(t)\right] d t+\ell\left(x^{*}(T)\right) \leq \liminf _{n \rightarrow+\infty} J\left(x_{\epsilon_{n}}, u_{\epsilon_{n}}\right) \\
& \leq \inf \left\{J(x, u):(x, u) \text { solves (1.2) and } x^{\prime} \in L^{\alpha}\left(I ; \mathbb{R}^{N}\right)\right\} .
\end{aligned}
$$

It follows that $v^{*}(t)=0$, for a.e. $t \in I$. So, by Proposition 2.3, $\left(x^{*}, u^{*}\right)$ minimizes (1.1) over all trajectory/control pairs $(x, u)$ solving (1.2). Moreover, $L\left(x^{*}(\cdot), u^{*}(\cdot)\right)$ is bounded in $L^{\infty}$, implying conclusions a).

Arguing as in the proof of Lemma 2.6, to obtain (A.20) and (A.21), we deduce that the optimal trajectory/control pair $\left(x^{*}, u^{*}\right)$ satisfies the Pontryagin Maximum Principle (see Vinter [12, p.203]). So, there exist $c \in \mathbb{R}$ and $p \in W^{1,1}\left(I ; \mathbb{R}^{N}\right)$ such that, for a.e. $t \in I$,

$c=\left\langle p(t), f\left(x^{*}(t), u^{*}(t)\right)\right\rangle-L\left(x^{*}(t), u^{*}(t)\right)=\max _{u \in U}\left\{\left\langle p(t), f\left(x^{*}(t), u\right)\right\rangle-L\left(x^{*}(t), u\right)\right\}$

and

$$
-p^{\prime}(t) \in\left(\partial_{x} f\right)^{*}\left(x^{*}(t), u^{*}(t)\right) p(t)-\partial_{x} L\left(x^{*}(t), u^{*}(t)\right) .
$$

Hence, by (H) i), for a.e. $t \in I$,

$$
\left|p^{\prime}(t)\right| \leq C_{k}\left[1+\left|f\left(x^{*}(t), u^{*}(t)\right)\right|+L\left(x^{*}(t), u^{*}(t)\right)^{1 / \alpha}\right]|p(t)|+C_{k}\left[1+L\left(x^{*}(t), u^{*}(t)\right)\right]
$$

and b) follows from conclusions a). 
Proof of Theorem 3.2. This proof follows the same ideas as the proof of Theorem 3.1. The only difference is in Step 1, when using assumption 2) in (3.6). Here, to apply assumption $\left.2^{\prime}\right)$, we have to know that there exists $\nu \geq \nu_{0}$ such that, for a.e. $t \in I$ and every $\epsilon \in\left(0, \epsilon_{0}\right)$, the elements $p_{\epsilon}(t)-\epsilon\left|f\left(x_{\epsilon}(t), u_{\epsilon}(t)\right)\right|^{\alpha-1} \phi_{\varepsilon}(t) \epsilon$ $P\left(x_{\epsilon}(t), u_{\epsilon}(t)\right)$ defined in (3.5) are bounded by $\nu$.

From Lemma 2.7, the functions $p_{\epsilon}$ defined in (3.4) satisfy

$$
\sup _{\epsilon \in\left(0, \epsilon_{0}\right)}\left\|p_{\epsilon}\right\|_{\infty} \leq M, \quad \text { for some } M>0 .
$$

We claim that

$$
c_{\epsilon} \geq-\gamma, \quad \text { for every } \epsilon \in\left(0, \epsilon_{0}\right) \text { and for some } \gamma>0 .
$$

Indeed, fix any $u_{0} \in U$ and let $t \in A_{\epsilon}$ be such that (3.4) holds true. Then

$$
c_{\epsilon} \geq\left\langle p_{\epsilon}(t), f\left(x_{\epsilon}(t), u_{0}\right)\right\rangle-L\left(x_{\epsilon}(t), u_{0}\right)-\frac{\epsilon}{\alpha}\left|f\left(x_{\epsilon}(t), u_{0}\right)\right|^{\alpha} .
$$

From (3.3) and (3.15), since $f\left(\cdot, u_{0}\right)$ and $L\left(\cdot, u_{0}\right)$ are continuous, (3.16) follows.

We next claim that, for every $\epsilon \in\left(0, \epsilon_{0}\right)$ and a.e. $t \in I$,

$$
\epsilon\left|f\left(x_{\epsilon}(t), u_{\epsilon}(t)\right)\right|^{\alpha-1} \leq \alpha M+\alpha \gamma+\epsilon_{0} .
$$

If $\left|f\left(x_{\epsilon}(t), u_{\epsilon}(t)\right)\right| \leq 1$, the above bound is trivial. Assume that $\left|f\left(x_{\epsilon}(t), u_{\epsilon}(t)\right)\right|>1$ and that (3.4) holds true at $t$. Since $L \geq 0$ and, from (3.4) and (3.16),

$$
c_{\epsilon}=\left\langle p_{\epsilon}(t), f\left(x_{\epsilon}(t), u_{\epsilon}(t)\right)\right\rangle-L\left(x_{\epsilon}(t), u_{\epsilon}(t)\right)-\frac{\epsilon}{\alpha}\left|f\left(x_{\epsilon}(t), u_{\epsilon}(t)\right)\right|^{\alpha} \geq-\gamma,
$$

applying (3.15) we obtain that

$$
\begin{aligned}
\epsilon\left|f\left(x_{\epsilon}(t), u_{\epsilon}(t)\right)\right|^{\alpha} & \leq \alpha M\left|f\left(x_{\epsilon}(t), u_{\epsilon}(t)\right)\right|+\alpha \gamma \\
& \leq \alpha M\left|f\left(x_{\epsilon}(t), u_{\epsilon}(t)\right)\right|+\alpha \gamma\left|f\left(x_{\epsilon}(t), u_{\epsilon}(t)\right)\right| .
\end{aligned}
$$

Dividing by $\left|f\left(x_{\epsilon}(t), u_{\epsilon}(t)\right)\right|$ we deduce (3.17).

From (3.15) and (3.17), taking $\nu=\max \left\{\nu_{0},(\alpha+1) M+\alpha \gamma+\epsilon_{0}\right\}$, we have that

$$
\left.\left|p_{\epsilon}(t)-\epsilon\right| f\left(x_{\epsilon}(t), u_{\epsilon}(t)\right)\right|^{\alpha-1} \phi_{\varepsilon}(t) \mid \leq \nu .
$$

So, we can apply assumption $\left.2^{\prime}\right)$ to obtain that there exists $c(k) \geq k$ such that

$$
\sup _{\substack{|x| \leq k,|f(x, u)| \leq k \\ p \in P_{\nu}(x, u)}} H(x, u, p)<\inf _{\substack{|x| \leq k,|f(x, u)| \geq c(k) \\ p \in P_{\nu}(x, u)}} H(x, u, p),
$$

and the proof follows as in Theorem 3.1 .

Remark 3.3. In Theorem 3.1, replace the assumptions on $\left(x_{1}, u_{1}\right)$ and assumption 1) with the following hypotheses, already having appeared in [5] in the context of the calculus of variations:

$\left(x_{1}, u_{1}\right)$ satisfies $x_{1}^{\prime} \in L^{\infty}\left(I ; \mathbb{R}^{N}\right)$ and $J\left(x_{1}, u_{1}\right)<+\infty$;

$\left.1^{\prime}\right)$ for any trajectory/control pair $(x, u)$ satisfying $x(0) \in C_{0}$ and $J(x, u) \leq$ $J\left(x_{1}, u_{1}\right)$,

$$
\|x\|_{\infty} \leq k \quad \text { and } \quad \operatorname{essinf}_{t \in I}|f(x(t), u(t))|<k .
$$

Then:

$\left.\mathrm{a}^{\prime}\right)$ problem (1.1)-(1.2) has an optimal solution $\left(x^{*}, u^{*}\right)$ such that $x^{*}$ is Lipschitzian;

$\left.\mathrm{b}^{\prime}\right)$ there exist $c \in \mathbb{R}$ and $p \in W^{1,1}\left(I ; \mathbb{R}^{N}\right)$ such that, for a.e. $t \in I$,

$$
c=\left\langle p(t), f\left(x^{*}(t), u^{*}(t)\right)\right\rangle-L\left(x^{*}(t), u^{*}(t)\right) .
$$


Indeed, if $\left(x_{1}, u_{1}\right)$ is optimal, then $\left.\mathrm{a}^{\prime}\right)$ holds true taking $\left(x^{*}, u^{*}\right)=\left(x_{1}, u_{1}\right)$. The validity of $\left.\mathrm{b}^{\prime}\right)$ follows from the Pontryagin Maximum Principle; see [12]. If $J\left(x_{1}, u_{1}\right)>$ $\inf \{J(x, u):(x, u)$ solves (1.2) $\}$, then working as in the proof of Theorem 3.1 we obtain $\left.\mathrm{a}^{\prime}\right)$ and $\left.\mathrm{b}^{\prime}\right)$.

\section{Applications}

In this section we provide some examples of problems which admit an optimal solution $\left(x^{*}, u^{*}\right)$ satisfying the DuBois-Reymond necessary optimality condition and such that $x^{*}$ is Lipschitzian and $L\left(x^{*}(\cdot), u^{*}(\cdot)\right)$ is essentially bounded. In the following examples $U$ will always be a closed convex cone. Observe that in this case:

$$
\text { for every } u \in U \text { and every } n \in N_{U}(u), \quad\langle n, u\rangle=0 .
$$

Example 4.1 (Superlinear growth with respect to dynamics). Consider the problem of minimizing the functional

$$
J(x, u)=\int_{0}^{T} L(x(t), u(t)) d t+\ell(x(T))
$$

over all pairs $(x, u)$ satisfying

$$
\begin{cases}x^{\prime}(t)=f(x(t))+B u(t) & \text { for a.e. } t \in I, \\ u(t) \in U & \text { for a.e. } t \in I \\ x(0)=x_{0}, & \end{cases}
$$

where $I=[0, T], L, \ell \geq 0, L(x, \cdot)$ is convex, $\forall x \in \mathbb{R}^{N}, x_{0} \in \mathbb{R}^{N}$ is fixed, and $B$ is an $n \times m$ matrix. Suppose that $f$ is globally Lipschitz with Lipschitz constant $C_{f}$, and that, $\forall R>0, \exists C_{R}>0$ such that, for every $x, y \in B(0, R)$ and every $u \in U$,

$|L(x, u)-L(y, u)| \leq C_{R}|x-y|[1+L(x, u) \wedge L(y, u)]$ and $|\ell(x)-\ell(y)| \leq C_{R}|x-y|$.

Furthermore, assume that there exists a function $\Phi: \mathbb{R} \rightarrow \mathbb{R}$ satisfying

$$
\lim _{r \rightarrow+\infty} \frac{\Phi(r)}{r}=+\infty \quad \text { and } \quad L(x, u) \geq \Phi(|f(x)+B u|), \quad \forall(x, u) \in \mathbb{R}^{N} \times \mathbb{R}^{m} .
$$

Consider the trajectory/control pair $(\bar{x}, \bar{u})$, where $\bar{u} \equiv 0$.

If $J(\bar{x}, \bar{u})=\inf \{J(x, u):(x, u)$ solves $(4.3)\}$, then $(\bar{x}, \bar{u})$ is the optimal solution we are looking for: by the assumptions on $L$ and $f, \bar{x}$ is Lipschitzian, $L(\bar{x}(\cdot), \bar{u}(\cdot))$ is essentially bounded, and the DuBois-Reymond necessary optimality conditions are satisfied; see [12].

Otherwise, we prove that the assumptions of Theorem 3.2 hold true taking $\left(x_{1}, u_{1}\right)=(\bar{x}, \bar{u})$. Indeed, to verify $\left(\mathrm{H}^{\prime}\right)$ notice that: i1), i2), i3') follow immediately from the assumptions on $L, \ell, f$; ii) follows from the Lipschitzianity of $f$; to prove iii) notice that the choice of $\bar{u}$ and the continuity of $L(\cdot, u)$ imply that, $\forall R>0, L(\cdot, 0)$ is bounded on $B(0, R)$; iv) comes from the fact that $L(x, \cdot)$ is convex and the dynamic is affine in the control. Using the superlinear growth and the Lipschitzianity of $f$, it is easy to verify assumption 1) of Theorem 3.1. Indeed, let $\left(x_{1}, u_{1}\right)=(\bar{x}, \bar{u})$ and let $(x, u)$ be a trajectory/control pair of (4.3) such that $J(x, u)<J(\bar{x}, \bar{u})$ and let $\gamma>0$ be such that

$$
|f(x)+B u| \leq \Phi(|f(x)+B u|), \quad \text { as }|f(x)+B u| \geq \gamma .
$$


Then, from (4.4),

$$
\begin{aligned}
\int_{0}^{T}|f(x(t))+B u(t)| d t & \leq T \gamma+\int_{0}^{T} \Phi(|f(x(t))+B u(t)|) d t \\
& \leq T \gamma+\int_{0}^{T} L(x(t), u(t)) d t+\ell(x(T))<T \gamma+J(\bar{x}, 0),
\end{aligned}
$$

so that, for some $k>0,\|x\|_{\infty} \leq k$ and $\operatorname{ess} \inf _{t \in I}|f(x(t))+B u(t)|<k$.

To show $\left.2^{\prime}\right)$, notice that if $p \in P(x, u)$, then $B^{*} p \in \partial_{u} L(x, u)+N_{U}(u)$. Hence, if $|p| \leq \nu$,

$$
\begin{aligned}
& \Phi(|f(x)+B u|) \leq L(x, u) \leq L(x, 0)+\left\langle B^{*} p, u\right\rangle=L(x, 0)+\langle p, B u\rangle \\
& \quad=L(x, 0)+\langle p, f(x)+B u\rangle-\langle p, f(x)\rangle \leq L(x, 0)+\langle p, f(x)+B u\rangle+\nu \sup _{|x| \leq k}|f(x)| .
\end{aligned}
$$

It follows that, when $c$ is large enough, the set

$$
\mathcal{C}_{c}=\{(x, u, p):|x| \leq k,|f(x)+B u| \geq c, p \in P(x, u),|p| \leq \nu\}
$$

is empty. So $\inf _{\mathcal{C}_{c}} H(x, u, p)=+\infty$ and $\left.2^{\prime}\right)$ follows.

Example 4.2 (Superlinear growth with respect to control). Consider problem (4.2)-(4.3) introduced in Example 4.1. Assume that there exists a function $\Phi$ : $\mathbb{R} \rightarrow \mathbb{R}$ satisfying

$$
\lim _{r \rightarrow+\infty} \frac{\Phi(r)}{r}=+\infty \quad \text { and } \quad L(x, u) \geq \Phi(|u|), \quad \forall(x, u) \in \mathbb{R}^{N} \times \mathbb{R}^{m} .
$$

As in the previous example, consider the trajectory/control pair $(\bar{x}, \bar{u})$, where $\bar{u} \equiv 0$. If $J(\bar{x}, \bar{u})=\inf \{J(x, u):(x, u)$ solves (4.3) $\}$, then $(\bar{x}, \bar{u})$ is the optimal solution we are looking for.

Otherwise, we prove that the assumptions of Theorem 3.2 hold true. The validity of $\left(\mathrm{H}^{\prime}\right)$ is proved by arguing as in Example 4.1. To prove 1) of Theorem 3.1, set $\left(x_{1}, u_{1}\right)=(\bar{x}, \bar{u})$ and consider a trajectory/control pair $(x, u)$ satisfying (4.3) and $J(x, u)<J(\bar{x}, \bar{u})$, and let $\gamma>0$ be such that

$$
|u| \leq \Phi(|u|), \quad \text { as }|u| \geq \gamma .
$$

Then, from the superlinear growth,

$$
\begin{aligned}
\int_{0}^{T}|u(t)| d t & \leq T \gamma+\int_{0}^{T} \Phi(|u(t)|) d t \\
& \leq T \gamma+\int_{0}^{T} L(x(t), u(t)) d t+\ell(x(T))<T \gamma+J(\bar{x}, \bar{u}) .
\end{aligned}
$$

So, since, for a.e. $t \in I,\left|x^{\prime}(t)\right| \leq C_{f}|x(t)|+|f(0)|+|| B|||u(t)|$, Gronwall's Lemma implies that, for some $k>0,\|x\|_{\infty} \leq k$ and $\operatorname{ess~inf}_{t \in I}|f(x(t))+B u(t)|<k$.

To show $\left.2^{\prime}\right)$, notice that if $p \in P(x, u)$, then $B^{*} p \in \partial_{u} L(x, u)+N_{U}(u)$. Hence, if $|p| \leq \nu$, then

$$
\Phi(|u|) \leq L(x, u) \leq L(x, 0)+\left\langle B^{*} p, u\right\rangle \leq L(x, 0)+\nu\left\|B^{*}\right\||u| .
$$

Defining $\mathcal{C}_{c}$ as in Example 4.1, we obtain that, for $c$ large, $\mathcal{C}_{c}=\emptyset$, and again $2^{\prime}$ ) holds true. 
Example 4.3 ( $\alpha$-growth with respect to control). Consider the problem of minimizing the functional

$$
J(x, u)=\int_{0}^{T} L(x(t), u(t)) d t+\ell(x(T))
$$

on measurable $u$ and absolutely continuous $x$ satisfying

$$
\begin{cases}x^{\prime}(t)=f(x(t))+g(x(t)) u(t) & \text { for a.e. } t \in I, \\ u(t) \in U & \text { for a.e. } t \in I, \\ x(0)=x_{0}, & \end{cases}
$$

where $I=[0, T], L, \ell \geq 0, L(x, \cdot)$ is convex, $\forall x \in \mathbb{R}^{N}, x_{0} \in \mathbb{R}^{N}$ is fixed, and $f, g$ are globally Lipschitz with Lipschitz constants, respectively, $C_{f}, C_{g}$. Suppose that, $\forall R>0, \exists C_{R}>0$ such that

$|L(x, u)-L(y, u)| \leq C_{R}|x-y|[1+L(x, u) \wedge L(y, u)]$ and $|\ell(x)-\ell(y)| \leq C_{R}|x-y|$, $\forall x, y \in B(0, R)$ and $\forall u \in U$. Assume there exist $\beta>0$ and $\alpha>1$ such that

$$
L(x, u) \geq \beta|u|^{\alpha} \quad \text { for all }(x, u) \in \mathbb{R}^{N} \times \mathbb{R}^{m} .
$$

Arguing as in the previous examples, if the trajectory/control pair $(\bar{x}, \bar{u})$, where $\bar{u} \equiv 0$, satisfies $J(\bar{x}, \bar{u})=\inf \{J(x, u):(x, u)$ solves (4.5) $\}$, then $(\bar{x}, \bar{u})$ is the optimal solution we are looking for.

Otherwise, the assumptions of Theorem 3.2 hold true. Indeed, to verify $\left(\mathrm{H}^{\prime}\right)$ notice that: i1) and i2) hold true since $L, \ell$ are locally Lipschitz and, for every $x, y \in \mathbb{R}^{N}$, we have

$$
\begin{aligned}
& |f(x)+g(x) u-f(y)-g(y) u| \leq|f(x)-f(y)|+|[g(x)-g(y)] u| \\
& \quad \leq C_{f}|x-y|+C_{g}|x-y||u| \leq|x-y|\left[C_{f}+\frac{C_{g}}{\beta^{1 / \alpha}}(L(x, u) \wedge L(y, u))^{1 / \alpha}\right] \\
& \leq C|x-y|\left[1+(L(x, u) \wedge L(y, u))^{1 / \alpha}\right]
\end{aligned}
$$

where $C=\max \left\{C_{f}, \frac{C_{g}}{\beta^{1 / \alpha}}\right\}$. So, i $\left.3^{\prime}\right)$ follows. To verify ii), iii) and iv) we proceed as in Example 4.1. Setting $\left(x_{1}, u_{1}\right)=(\bar{x}, \bar{u})$, arguing as in Examples 4.1 and 4.2 , and taking into account the global Lipschitzianity of $g$, we obtain the validity of assumption 1) of Theorem 3.2. To show $\left.2^{\prime}\right)$, notice that if $p \in P_{\nu}(x, u)$, then $g(x)^{*} p \in \partial_{u} L(x, u)+N_{U}(u)$. Hence,

$$
\beta|u|^{\alpha} \leq L(x, u) \leq L(x, 0)+\left\langle g(x)^{*} p, u\right\rangle \leq L(x, 0)+\nu\left\|g(x)^{*}\right\||u| .
$$

It follows that, when $c$ is large enough, the set $\mathcal{C}_{c}$ defined as in Example 4.1 with $B$ replaced by $g(x)$ is empty. So, $\left.2^{\prime}\right)$ follows.

Remark 4.4. The problems in Examples 4.1, 4.2, and 4.3 also satisfy the assumptions of Theorem 3.1 under the hypothesis that $f$ and $g$ are locally Lipschitz, jointly with some growth assumption as, for instance,

$$
\exists a>0 \text { such that, } \forall x \in \mathbb{R}^{N}, \quad|f(x)|+|g(x)| \leq a(|x|+1),
$$

instead of globally Lipschitz.

Example 4.5 (Slow growth). Consider the problem of minimizing the functional

$$
J(x, y, u, v)=\int_{0}^{1} \sqrt{1+u(t)^{2}+v(t)^{2}} d t+x(1)^{2}+y(1)^{2}
$$


on measurable $(u, v)$ and absolutely continuous $(x, y)$ satisfying

$$
\begin{cases}x^{\prime}(t)=x(t) u(t)+y(t) v(t) & \text { for a.e. } t \in I, \\ y^{\prime}(t)=u(t)+v(t) & \text { for a.e. } t \in I, \\ u(t), v(t) \in[0,+\infty) & \text { for a.e. } t \in I \\ x(0)=x_{0} \in \mathbb{R}, y(0)=y_{0} \geq 0, & \end{cases}
$$

where $I=[0,1]$. We first check that the assumptions of Theorem 3.1 are satisfied. Assumptions $(\mathrm{H})$ hold true. Indeed, to prove i) notice that $L$ is independent of the trajectory, and, since $u, v \geq 0$, for every $(x, y)$,

$$
\left|\partial_{(x, y)} f(x, y, u, v)\right|=\sqrt{u^{2}+v^{2}} \leq \sqrt{(x u+y v)^{2}+(u+v)^{2}}=|f(x, y, u, v)| .
$$

Taking $\bar{u} \equiv \bar{v} \equiv 0$ and arguing as in the previous examples, we deduce the validity of ii), iii) and iv). We wish to show assumptions 1) and 2) of Theorem 3.1. Set $\left(u_{1}, v_{1}\right) \equiv(1,0)$, so that, $\forall t \in I,\left(x_{1}(t), y_{1}(t)\right)=\left(x_{0} e^{t}, y_{0}+t\right)$ and $J\left(x_{1}, y_{1}, u_{1}, v_{1}\right)=\sqrt{2}+x_{0}^{2} e^{2}+\left(y_{0}+1\right)^{2}>1+x_{0}^{2}+y_{0}^{2}=J(\bar{x}, \bar{y}, \bar{u}, \bar{v})$. Let $(x, y, u, v)$ be a trajectory/control pair such that

$$
\int_{0}^{1} \sqrt{1+u(t)^{2}+v(t)^{2}} d t+x(1)^{2}+y(1)^{2}<\sqrt{2}+x_{0}^{2} e^{2}+y_{0}^{2}=k_{0} .
$$

Since $y_{0} \geq 0$ and $u, v \geq 0, y$ is nonnegative and nondecreasing, so, by (4.7),

$$
\|y\|_{\infty} \leq y(1)<\sqrt{k_{0}} .
$$

Moreover, since, for a.e. $t \in I,\left|x^{\prime}(t)\right| \leq|x(t)| u(t)+y(t) v(t)$, from Gronwall's Lemma, (4.7), and the estimate on $\|y\|_{\infty}$, we obtain that

$$
\begin{aligned}
\|x\|_{\infty} & \leq e^{\int_{0}^{1} u(t) d t}\left[\left|x_{0}\right|+\int_{0}^{1} y(t) v(t) d t\right] \leq e^{\|y\|_{\infty}}\left[\left|x_{0}\right|+\|y\|_{\infty} \int_{0}^{1} v(t) d t\right] \\
& \leq e^{\|y\|_{\infty}}\left(\left|x_{0}\right|+\|y\|_{\infty}^{2}\right) \leq e^{\sqrt{k_{0}}}\left(\sqrt{k_{0}}+k_{0}\right)=k_{1} .
\end{aligned}
$$

So,

$$
\|(x, y)\|_{\infty} \leq \sqrt{k_{1}^{2}+k_{0}}
$$

From (4.8),

$$
\begin{aligned}
\int_{0}^{1}|f(x(t), y(t), u(t), v(t))| d t & \leq \int_{0}^{1} \sqrt{\left[(x(t)+y(t))^{2}+1\right](u(t)+v(t))^{2}} d t \\
& \leq\|y\|_{\infty} \sqrt{2\|(x, y)\|_{\infty}^{2}+1} \leq \sqrt{k_{0}} \sqrt{2\left(k_{1}^{2}+k_{0}\right)+1}
\end{aligned}
$$

Taking $\left.k=\max \left\{\sqrt{k_{1}^{2}+k_{0}}, \sqrt{k_{0}} \sqrt{2\left(k_{1}^{2}+k_{0}\right)+1}\right\}, 1\right)$ holds. To prove 2), observe first that, setting

$$
f_{0}(x, y)=\left[\begin{array}{ll}
x & y \\
1 & 1
\end{array}\right]
$$

we have that, for every $(x, y, u, v) \in \mathbb{R}^{2} \times U$, with $U=\left\{(u, v) \in \mathbb{R}^{2}: u, v \geq 0\right\}$,

$$
f(x, y, u, v)=f_{0}(x, y)\left[\begin{array}{l}
u \\
v
\end{array}\right] \quad \text { and } \quad \partial_{(u, v)} f(x, y, u, v)=f_{0}(x, y) \text {. }
$$

So, if $p \in P(x, y, u, v)$, then

$$
f_{0}^{*}(x, y) p \in \partial_{(u, v)} L(u, v)+N_{U}(u, v),
$$


and, since $U$ is a cone, from (4.1) and (4.10) we obtain that

$$
\begin{aligned}
& H(x, y, u, v, p)=\langle p, f(x, y, u, v)\rangle-L(u, v)=\left\langle f_{0}^{*}(x, y) p,(u, v)\right\rangle-L(u, v) \\
& \quad=\left\langle\partial_{(u, v)} L(u, v),(u, v)\right\rangle-L(u, v)=\frac{1}{\sqrt{1+u^{2}+v^{2}}}|(u, v)|^{2}-\sqrt{1+u^{2}+v^{2}} \\
& \quad=-\frac{1}{\sqrt{1+u^{2}+v^{2}}} .
\end{aligned}
$$

Since, $\forall u, v \geq 0, u^{2}+v^{2} \leq|f(x, y, u, v)|^{2}$ and the function $s \mapsto-1 /(1+s)$ is increasing,

$$
\sup _{\substack{|(x, y)| \leq k,|f(x, y, u, v)| \leq k \\ p \in P(x, y, u, v)}} H(x, y, u, v, p) \leq \sup _{\substack{|(x, y)| \leq k \\|f(x, y, u, v)| \leq k}} \frac{-1}{\sqrt{1+u^{2}+v^{2}}} \leq \frac{-1}{1+k^{2}} .
$$

Moreover, since $|f(x, y, u, v)|^{2} \leq\left[(x+y)^{2}+1\right](u+v)^{2} \leq\left(2|(x, y)|^{2}+1\right) 2\left(u^{2}+v^{2}\right)$, $\forall c>0$,

$$
\begin{aligned}
\inf _{\substack{|(x, y)| \leq k,|f(x, y, u, v)| \geq c \\
p \in P(x, y, u, v)}} H(x, y, u, v, p) & \geq \inf _{\substack{|(x, y)| \leq k \\
|f(x, y, u, v)| \geq c}} \frac{-1}{\sqrt{1+u^{2}+v^{2}}} \\
& \geq \frac{-1}{\sqrt{1+c^{2} /\left(4 k^{2}+2\right)}} .
\end{aligned}
$$

Since $\frac{-1}{\sqrt{1+c^{2} /\left(4 k^{2}+2\right)}} \rightarrow 0$, as $c \rightarrow+\infty$, for $c$ sufficiently large we obtain the desired inequality. So, 2) also holds.

Example 4.6 (No growth). Consider the problem of minimizing the functional

$$
J(x, u)=\int_{0}^{1} e^{-[u(t)+2 v(t)]} d t+x(1)^{2}+y(1)^{2}
$$

on measurable $(u, v)$ and absolutely continuous $(x, y)$ satisfying (4.6). We prove that the assumptions of Theorem 3.1 are satisfied. Taking $\bar{u} \equiv \bar{v} \equiv 0$ and arguing as in the previous example we deduce the validity of $(\mathrm{H})$. Set $\left(u_{1}, v_{1}\right) \equiv(1,0)$, so that, $\forall t \in I,\left(x_{1}(t), y_{1}(t)\right)=\left(x_{0} e^{t}, y_{0}+t\right)$ and $J\left(x_{1}, y_{1}, u_{1}, v_{1}\right)=e^{-1}+x_{0}^{2} e^{2}+\left(y_{0}+1\right)^{2}>$ $1+x_{0}^{2}+y_{0}^{2}=J(\bar{x}, \bar{y}, \bar{u}, \bar{v})$. Let $(x, y, u, v)$ be a trajectory/control pair such that

$$
\int_{0}^{1} e^{-[u(t)+2 v(t)]} d t+x(1)^{2}+y(1)^{2}<e^{-1}+x_{0}^{2} e^{2}+\left(y_{0}+1\right)^{2}=k_{0} .
$$

Exactly as in Example 4.5 we check that 1) of Theorem 3.1 holds true. We deduce from (4.9) and (4.10) that, if $p \in P(x, y, u, v)$, then $f_{0}^{*}(x, y) p \in \partial_{(u, v)} L(u, v)+$ $N_{U}(u, v)$, and, since $U$ is a cone, from (4.1) and (4.10) we obtain that

$$
\begin{aligned}
H(x, y, u, v, p) & =\langle p, f(x, y, u, v)\rangle-L(u, v)=\left\langle f_{0}^{*}(x, y) p,(u, v)\right\rangle-L(u, v) \\
& =\left\langle\partial_{(u, v)} L(u, v),(u, v)\right\rangle-L(u, v)=-e^{-(u+2 v)}(u+2 v)-e^{-(u+2 v)} \\
& =-e^{-(u+2 v)}(1+u+2 v) .
\end{aligned}
$$

Since, $\forall u, v \geq 0, u+2 v \leq 2|f(x, y, u, v)|$ and the function $s \mapsto \frac{-(1+s)}{e^{s}}$ is increasing,

$$
\sup _{\substack{|(x, y)| \leq k,|f(x, y, u, v)| \leq k \\ p \in P(x, y, u, v)}} H(x, y, u, v, p) \leq \sup _{\substack{|(x, y)| \leq k \\|f(x, y, u, v)| \leq k}}-\frac{(1+u+2 v)}{e^{(u+2 v)}} \leq-\frac{(1+2 k)}{e^{2 k}} .
$$


Moreover, since, $\forall u, v \geq 0,|f(x, y, u, v)| \leq 2(u+2 v) \sqrt{1+2|(x, y)|^{2}}$, we have $\forall c>0$,

$$
\begin{aligned}
\inf _{\substack{|(x, y)| \leq k,|f(x, y, u, v)| \geq c \\
p \in P(x, y, u, v)}} H(x, y, u, v, p) \geq & \inf _{\substack{|(x, y)| \leq k \\
|f(x, y, u, v)| \geq c}}-\frac{(1+u+2 v)}{e^{(u+2 v)}} \\
\geq & -\frac{1+c /\left(2 \sqrt{1+2 k^{2}}\right)}{e^{c /\left(2 \sqrt{1+2 k^{2}}\right)}} .
\end{aligned}
$$

Since $-\frac{1+c /\left(2 \sqrt{1+2 k^{2}}\right)}{e^{c /\left(2 \sqrt{1+2 k^{2}}\right)}} \rightarrow 0$, as $c \rightarrow+\infty$, for $c$ sufficiently large we obtain the desired inequality. So, 2) also holds true.

\section{Appendix A. Proofs of Lemma 2.6 and Lemma 2.7}

Proof of Lemma 2.6. Let $\epsilon>0$ and let $\beta_{\epsilon}$ be the infimum of problem (2.5), (1.2). From Proposition 2.3 and by the assumptions on $\left(x_{1}, u_{1}\right)$ we have that

$$
\begin{aligned}
J\left(x_{1}, u_{1}\right) & >\inf \left\{J(x, u):(x, u) \text { solves (1.2) and } x^{\prime} \in L^{1}\left(I ; \mathbb{R}^{N}\right)\right\} \\
& =\inf \left\{J(x, u):(x, u) \text { solves (1.2) and } x^{\prime} \in L^{\alpha}\left(I ; \mathbb{R}^{N}\right)\right\} .
\end{aligned}
$$

So, there exists a trajectory/control pair $(\tilde{x}, \tilde{u})$ such that $\tilde{x}^{\prime} \in L^{\alpha}\left(I ; \mathbb{R}^{N}\right), \tilde{x}(0) \in C_{0}$ and $J(\tilde{x}, \tilde{u})<J\left(x_{1}, u_{1}\right)$. Since $f(\tilde{x}(\cdot), \tilde{u}(\cdot)) \in L^{\alpha}\left(I ; \mathbb{R}^{N}\right)$, for $\epsilon$ sufficiently small (say $\left.\epsilon \in\left(0, \epsilon_{0}\right)\right)$, we have

$$
\beta_{\epsilon} \leq J_{\epsilon}(\tilde{x}, \tilde{u})<J\left(x_{1}, u_{1}\right)
$$

Fix $\epsilon \in\left(0, \epsilon_{0}\right)$.

To prove a) we show that there exist $\left\{\left(x_{n}, u_{n}\right)\right\}_{n \in \mathbb{N}}$ in $W^{1,1}\left(I ; \mathbb{R}^{N}\right) \times \mathcal{U}$ satisfying (1.2), $x_{\epsilon} \in W^{1,1}\left(I ; \mathbb{R}^{N}\right)$, and $\xi \in L^{1}(I ; \mathbb{R})$ such that, when $n \rightarrow+\infty$,

$$
J_{\epsilon}\left(x_{n}, u_{n}\right) \rightarrow \beta_{\epsilon} \quad \text { and } \quad \begin{cases}x_{n} \rightarrow x_{\epsilon} & \text { uniformly, } \\ x_{n}^{\prime} \rightarrow x_{\epsilon}{ }^{\prime} & \text { weakly in } L^{1}, \\ L_{\epsilon}\left(x_{n}(\cdot), u_{n}(\cdot)\right) \rightarrow \xi & \text { weakly in } L^{1} .\end{cases}
$$

Then, we prove that there exists a measurable function $u_{\epsilon}: I \rightarrow \mathbb{R}^{m}$ such that the trajectory/control pair $\left(x_{\epsilon}, u_{\epsilon}\right)$ satisfies (1.2) and $J_{\epsilon}\left(x_{\epsilon}, u_{\epsilon}\right)=\beta_{\epsilon}$.

Step 1. Fix $x_{0} \in C_{0}$ and define

$$
\tilde{J}_{\epsilon}(u)=\int_{0}^{T} L_{\epsilon}(x(t), u(t)) d t+\ell(x(T))
$$

where $x \in W^{1,1}\left(I ; \mathbb{R}^{N}\right)$ solves the system

$$
\begin{cases}x^{\prime}(t)=f(x(t), u(t)) & \text { for a.e. } t \in I \\ u(t) \in U & \text { for a.e. } t \in I \\ x(0)=x_{0} & \end{cases}
$$

We set $\tilde{J}_{\epsilon}(u)=+\infty$ if there is no solution $x$ for such $u$ defined on $I$, or if $L_{\epsilon}(x(\cdot), u(\cdot))$ is not integrable on $I$. For every $u, v \in \mathcal{U}$, set

$$
d(u, v)=\mu(\{t \in I: u(t) \neq v(t)\}) .
$$

Then $d$ is a distance and $(\mathcal{U}, d)$ is a complete metric space; see [6, p. 202]. We claim that $\tilde{J}_{\epsilon}: \mathcal{U} \rightarrow \mathbb{R}$ is a lower semicontinuous functional; i.e., whenever 
$\lim _{n \rightarrow+\infty} d\left(u_{n}, u\right)=0$, then

$$
\tilde{J}_{\epsilon}(u) \leq \liminf _{n \rightarrow+\infty} \tilde{J}_{\epsilon}\left(u_{n}\right) .
$$

Indeed, let $u,\left\{u_{n}\right\}_{n \in \mathbb{N}}$ be elements of $\mathcal{U}$ such that $\lim _{n \rightarrow+\infty} d\left(u_{n}, u\right)=0$.

If $\liminf \operatorname{in}_{n \rightarrow+\infty} \tilde{J}_{\epsilon}\left(u_{n}\right)=+\infty$ the claim is obvious. Suppose that there exists $c>0$ such that $\liminf \operatorname{in}_{n \rightarrow+\infty} \tilde{J}_{\epsilon}\left(u_{n}\right)=c$. Taking a subsequence and keeping the same notation, we may suppose that $\lim _{n \rightarrow+\infty} \tilde{J}_{\epsilon}\left(u_{n}\right)=c$ and that, $\forall n \in \mathbb{N}$, $\tilde{J}_{\epsilon}\left(u_{n}\right) \leq c+1$, so that $u_{n} \in \operatorname{dom} \tilde{J}_{\epsilon}$, and $\exists x_{n} \in W^{1,1}\left(I ; \mathbb{R}^{N}\right)$ such that $\left(x_{n}, u_{n}\right)$ satisfies (A.2). Notice that the sequence $\left\{f\left(x_{n}(\cdot), u_{n}(\cdot)\right)\right\}_{n \in \mathbb{N}}$ is bounded in $L^{\alpha}$. By Lemma 2.5 there exists $x \in W^{1,1}\left(I ; \mathbb{R}^{N}\right)$ such that $x(0)=x_{0}$, and (taking a subsequence of $\left\{x_{n}\right\}_{n \in \mathbb{N}}$ and keeping the same notation),

$$
\text { as } n \rightarrow+\infty, \quad \begin{cases}x_{n} \rightarrow x & \text { uniformly, } \\ x_{n}^{\prime} \rightarrow x^{\prime} & \text { weakly in } L^{1} .\end{cases}
$$

We have to prove that $(x, u)$ solves (A.2). To do so we need to show that, for a.e. $t \in I, x^{\prime}(t)=f(x(t), u(t))$. Let $R>0$ be such that $x_{n}(t), x(t) \in B(0, R), \forall n \in \mathbb{N}$ and $\forall t \in I$. Set

$$
B_{n}=\left\{t \in I: u_{n}(t) \neq u(t)\right\} .
$$

Since $\lim _{n \rightarrow+\infty} \mu\left(B_{n}\right)=0$, there exists an increasing subsequence $\left\{n_{i}\right\}_{i \in \mathbb{N}}$ such that $\mu\left(B_{n_{i}}\right) \leq 1 /\left(2^{i+1}\right)$, so that $\mu\left(\bigcup_{i \geq k} B_{n_{i}}\right) \leq 1 / 2^{k}$. For $k \in \mathbb{N}$, set

$$
A_{k}=I \backslash\left(\bigcup_{i \geq k} B_{n_{i}}\right) \text {. }
$$

We have that $A_{k}=\left\{t \in I: u(t)=u_{n_{i}}(t), \forall i \geq k\right\}$ and $A_{k} \subset A_{k+1}$. Moreover,

$$
\text { since } \quad \mu\left(A_{k}\right) \geq \mu(I)-1 / 2^{k}, \quad \text { it follows that } \quad \lim _{k \rightarrow+\infty} \mu\left(A_{k}\right)=\mu(I) \text {. }
$$

Fix $k$. We have that, for $i \geq k$ and for a.e. $t \in I$,

$$
f\left(x_{n_{i}}(t), u(t)\right) \chi_{A_{k}}(t)=f\left(x_{n_{i}}(t), u_{n_{i}}(t)\right) \chi_{A_{k}}(t) .
$$

Let $\zeta \in L^{\infty}\left(I ; \mathbb{R}^{N}\right)$ and $\|\zeta\|_{\infty} \leq 1$. From (A.3) and (A.4) we obtain

$$
\begin{aligned}
\lim _{i \rightarrow+\infty} \int_{A_{k}} \zeta(t) f\left(x_{n_{i}}(t), u(t)\right) d t & =\lim _{i \rightarrow+\infty} \int_{A_{k}} \zeta(t) f\left(x_{n_{i}}(t), u_{n_{i}}(t)\right) d t \\
& =\int_{A_{k}} \zeta(t) x^{\prime}(t) d t .
\end{aligned}
$$

Since the sequence $\left\{f\left(x_{n_{i}}(\cdot), u_{n_{i}}(\cdot)\right)\right\}_{i \in \mathbb{N}}$ is bounded in $L^{\alpha}$, from A.4 we deduce that there exists $C>0$ such that, for every $i \in \mathbb{N}$,

$$
\left\|f\left(x_{n_{i}}(\cdot), u(\cdot)\right) \chi_{A_{k}}\right\|_{L^{\alpha}} \leq C .
$$

We claim that $\left\{\zeta f\left(x_{n_{i}}(\cdot), u(\cdot)\right) \chi_{A_{k}}\right\}_{i \in \mathbb{N}}$ is equi-integrable. Indeed, let $\delta>0$. For $\delta^{\prime}>0$ sufficiently small, $\forall E \subset I$ with measure $m(E) \leq \delta^{\prime}$, and $\forall i \in \mathbb{N}$, from (A.6) and Hölder's inequality we obtain that

$$
\begin{aligned}
& \int_{E}\left|\zeta(t) f\left(x_{n_{i}}(t), u(t)\right) \chi_{A_{k}}(t)\right| d t \\
& \leq\left(\int_{I}\left|\chi_{E}(t) \zeta(t)\right|^{\alpha^{\prime}} d t\right)^{1 / \alpha^{\prime}}\left(\int_{I}\left|f\left(x_{n_{i}}(t), u(t)\right) \chi_{A_{k}}(t)\right|^{\alpha} d t\right)^{1 / \alpha} \leq m(E)^{1 / \alpha^{\prime}} C \leq \delta .
\end{aligned}
$$


Since $f(\cdot, u)$ is continuous, the equi-integrability of $\left\{\zeta f\left(x_{n_{i}}(\cdot), u(\cdot)\right) \chi_{A_{k}}\right\}_{i \in \mathbb{N}}$ and (A.3) imply

$$
\lim _{i \rightarrow+\infty} \int_{A_{k}} \zeta(t) f\left(x_{n_{i}}(t), u(t)\right) d t=\int_{A_{k}} \zeta(t) f(x(t), u(t)) d t
$$

see [8, Theorem 9, p.325]. Applying (A.5) we deduce that

$$
\int_{A_{k}} \zeta(t) f(x(t), u(t)) d t=\int_{A_{k}} \zeta(t) x^{\prime}(t) d t
$$

So, $x^{\prime}(t)=f(x(t), u(t))$, for a.e. $t \in A_{k}$. Since this is true for every $k$ and $I=\left(\bigcup_{k \in \mathbb{N}} A_{k}\right) \cup \mathcal{N}$, with $\mu(\mathcal{N})=0$, it follows that $x^{\prime}(t)=f(x(t), u(t))$, for a.e. $t \in I$. We have that, $\forall k$,

$$
\begin{aligned}
\int_{A_{k}} & {\left[L\left(x_{n}(t), u_{n}(t)\right)+\frac{\epsilon}{\alpha}\left|f\left(x_{n}(t), u_{n}(t)\right)\right|^{\alpha}\right] d t+\ell\left(x_{n}(T)\right) } \\
& \geq \int_{A_{k}}\left[L\left(x_{n}(t), u(t)\right)+\frac{\epsilon}{\alpha}\left|f\left(x_{n}(t), u(t)\right)\right|^{\alpha}\right] d t+\ell(x(T))-C_{R}\left|x_{n}(T)-x(T)\right| .
\end{aligned}
$$

From the continuity of $L(\cdot, u)$ and $f(\cdot, u)$, applying Fatou's Lemma, we obtain

$$
\begin{aligned}
\liminf _{n \rightarrow+\infty} \tilde{J}_{\epsilon}\left(u_{n}\right) & \geq \liminf _{n \rightarrow+\infty} \int_{A_{k}}\left[L\left(x_{n}(t), u(t)\right)+\frac{\epsilon}{\alpha}\left|f\left(x_{n}(t), u(t)\right)\right|^{\alpha}\right] d t+\ell(x(T)) \\
& \geq \int_{A_{k}} \liminf _{n \rightarrow+\infty}\left[L\left(x_{n}(t), u(t)\right)+\frac{\epsilon}{\alpha}\left|f\left(x_{n}(t), u(t)\right)\right|^{\alpha}\right] d t+\ell(x(T)) \\
& =\int_{A_{k}}\left[L(x(t), u(t))+\frac{\epsilon}{\alpha}|f(x(t), u(t))|^{\alpha}\right] d t+\ell(x(T)) .
\end{aligned}
$$

Since this is true for every $k$, we deduce that $\liminf _{n \rightarrow+\infty} \tilde{J}_{\epsilon}\left(u_{n}\right) \geq \tilde{J}_{\epsilon}(u)$, whence the lower semicontinuity of $\tilde{J}_{\epsilon}$ follows.

Step 2. Let $\left(\bar{x}_{n}, \bar{u}_{n}\right)$ satisfy (1.2), for every $n \in \mathbb{N}$, and be such that

$$
\lim _{n \rightarrow+\infty} J_{\epsilon}\left(\bar{x}_{n}, \bar{u}_{n}\right)=\beta_{\epsilon},
$$

where $\beta_{\epsilon}$ is the infimum of problem (2.5), (1.2). Consider $\delta_{n}>0$ such that $\lim _{n \rightarrow+\infty} \delta_{n}=0$ and $J_{\epsilon}\left(\bar{x}_{n}, \bar{u}_{n}\right) \leq \beta_{\epsilon}+\delta_{n}$.

Fix $n$. Take $x_{0}=\bar{x}_{n}(0)$ and define $\tilde{J}_{\epsilon}$ as in step 1. Applying Ekeland's Theorem, [12], to $\tilde{J}_{\epsilon}$, we obtain that there exists $u_{n} \in \operatorname{dom} \tilde{J}_{\epsilon}$ satisfying $d\left(\bar{u}_{n}, u_{n}\right) \leq \sqrt{\delta_{n}}$ which is a minimizer for the lower semicontinuous functional

$$
J_{\epsilon}^{n}(u)=\tilde{J}_{\epsilon}(u)+\sqrt{\delta_{n}} d\left(u, u_{n}\right)
$$

Thus

$$
J_{\epsilon}^{n}\left(u_{n}\right)=\inf _{u \in \mathcal{U}} J_{\epsilon}^{n}(u) .
$$

Let $x_{n}: I \rightarrow \mathbb{R}^{N}$ be the solution to

$$
\left\{\begin{array}{l}
x^{\prime}(t)=f\left(x(t), u_{n}(t)\right) \quad \text { for a.e. } t \in I, \\
x(0)=\bar{x}_{n}(0) .
\end{array}\right.
$$

Notice that $\lim _{n \rightarrow+\infty} J_{\epsilon}\left(x_{n}, u_{n}\right)=\beta_{\epsilon}$, so that from (A.1) and the assumptions of Lemma 2.6. for any $n$ sufficiently large, $J\left(x_{n}, u_{n}\right) \leq J_{\epsilon}\left(x_{n}, u_{n}\right)<J\left(x_{1}, u_{1}\right)$ and $\left\|x_{n}\right\|_{\infty} \leq k$. Applying Lemma 2.5, taking a subsequence and keeping the same 
notation, it follows that there exists $x_{\epsilon} \in W^{1,1}\left(I ; \mathbb{R}^{N}\right)$ such that $x_{\epsilon}(0) \in C_{0}$ and that, when $n \rightarrow+\infty$,

$$
\begin{cases}x_{n} \rightarrow x_{\epsilon}, & \text { uniformly, } \\ x_{n}^{\prime} \rightarrow x_{\epsilon}^{\prime} & \text { weakly in } L^{1} .\end{cases}
$$

Step 3. We claim that there exists $\xi \in L^{1}\left(I ; \mathbb{R}^{N}\right)$ such that a subsequence

$$
L_{\epsilon}\left(x_{n_{i}}(\cdot), u_{n_{i}}(\cdot)\right) \rightarrow \xi \quad \text { weakly in } L^{1} .
$$

Since the sequence $\left\{L_{\epsilon}\left(x_{n}(\cdot), u_{n}(\cdot)\right)\right\}_{n \in \mathbb{N}}$ is bounded in $L^{1}(I ; \mathbb{R})$, to prove our claim we have to show that it is equi-integrable.

Fix $n \in \mathbb{N}$. Let $\bar{u}$ be as in assumption (H) ii) and let $t_{0} \in I$ be a Lebesgue point of

$$
L_{\epsilon}\left(x_{n}(\cdot), \bar{u}(\cdot)\right)-L_{\epsilon}\left(x_{n}(\cdot), u_{n}(\cdot)\right) \quad \text { and } \quad\left|f\left(x_{n}(\cdot), u_{n}(\cdot)\right)-f\left(x_{n}(\cdot), \bar{u}(\cdot)\right)\right|
$$

such that $x_{n}^{\prime}\left(t_{0}\right)=f\left(x_{n}\left(t_{0}\right), u_{n}\left(t_{0}\right)\right)$. The set of such points has full measure in $I$. For all small $h>0$, consider the controls

$$
u_{n}^{h}(t)= \begin{cases}u_{n}(t) & \text { if } t \leq t_{0}-h \\ \bar{u}(t) & \text { if } t_{0}-h<t<t_{0} \\ u_{n}(t) & \text { if } t \geq t_{0}\end{cases}
$$

We prove that, for all small $h>0$, there exists $x_{n}^{h} \in W^{1,1}\left(I ; \mathbb{R}^{N}\right)$ solving the system

$$
\left\{\begin{array}{l}
x^{\prime}(t)=f\left(x(t), u_{n}^{h}(t)\right) \\
x(0)=\bar{x}_{n}(0)
\end{array}\right.
$$

We have that, on $\left[0, t_{0}-h\right], x_{n}$ solves (A.8). Consider the system

$$
\left\{\begin{array}{l}
x^{\prime}(t)=f(x(t), \bar{u}(t)), \\
x\left(t_{0}-h\right)=x_{n}\left(t_{0}-h\right) .
\end{array}\right.
$$

Owing to the sublinear growth condition in $(\mathrm{H})$ ii), we conclude that there exists $y_{n}^{h} \in W^{1,1}\left(\left[t_{0}-h, t_{0}\right] ; \mathbb{R}^{N}\right)$ solving (A.9) on $\left[t_{0}-h, t_{0}\right]$. Let us consider the system

$$
\left\{\begin{array}{l}
x^{\prime}(t)=f\left(x(t), u_{n}(t)\right) \\
x\left(t_{0}\right)=y_{n}^{h}\left(t_{0}\right) .
\end{array}\right.
$$

We have to prove that, for all small $h>0$, there exists an absolutely continuous function $z_{n}^{h}:\left[t_{0}, T\right] \rightarrow \mathbb{R}^{N}$ solving (A.10) on $\left[t_{0}, T\right]$. Let us remark that for every $\delta>0$ there exists $\delta^{\prime}>0$ such that, for every $h \leq \delta^{\prime}$ and every $n$,

$$
\left|x_{n}\left(t_{0}\right)-y_{n}^{h}\left(t_{0}\right)\right| \leq \delta .
$$

Indeed, we have that

$$
\left|x_{n}\left(t_{0}\right)-y_{n}^{h}\left(t_{0}\right)\right| \leq \int_{t_{0}-h}^{t_{0}}\left(\left|f\left(x_{n}(s), u_{n}(s)\right)-f\left(y_{n}^{h}(s), \bar{u}(s)\right)\right|\right) d s .
$$

Now, recalling Lemma 2.5. we have that $\left\{f\left(x_{n}(\cdot), u_{n}(\cdot)\right)\right\}_{n \in \mathbb{N}}$ is equi-integrable in $I$. Thus, using assumption $(\mathrm{H})$ ii) the claim follows.

Fix $h>0$ and define, for $t \in\left[t_{0}, T\right]$,

$$
z(t)=y_{n}^{h}\left(t_{0}\right)+\int_{t_{0}}^{t} f\left(x_{n}(s), u_{n}(s)\right) d s .
$$


We have that $z \in W^{1,1}\left(\left[t_{0}, T\right] ; \mathbb{R}^{N}\right)$ and, for a.e. $t \in\left[t_{0}, T\right], z^{\prime}(t)=f\left(x_{n}(t), u_{n}(t)\right)$. Let $R>0$ and $\bar{h}>0$ be such that, $\forall t \in\left[t_{0}, T\right]$ and $\forall h \leq \bar{h}, x_{n}(t), z(t) \in B(0, R)$. Set

to obtain

$$
\gamma(t)=\left|z^{\prime}(t)-f\left(z(t), u_{n}(t)\right)\right|
$$

$$
\begin{aligned}
\gamma(t) & \leq C_{R}\left|x_{n}(t)-z(t)\right|\left[1+\left|f\left(x_{n}(t), u_{n}(t)\right)\right|+L\left(x_{n}(t), u_{n}(t)\right)^{1 / \alpha}\right] \\
& =C_{R}\left|x_{n}\left(t_{0}\right)-y_{n}^{h}\left(t_{0}\right)\right|\left[1+\left|f\left(x_{n}(t), u_{n}(t)\right)\right|+L\left(x_{n}(t), u_{n}(t)\right)^{1 / \alpha}\right] .
\end{aligned}
$$

Since $\lim _{h \rightarrow 0}\left|x_{n}\left(t_{0}\right)-y_{n}^{h}\left(t_{0}\right)\right|=0$ and, for some $C>0$,

$$
\int_{I}\left[1+\left|f\left(x_{n}(t), u_{n}(t)\right)\right|+L\left(x_{n}(t), u_{n}(t)\right)^{1 / \alpha}\right] d t \leq C
$$

for $h$ sufficiently small and some $C_{1}>0$, we have that

$$
\begin{aligned}
& \int_{t_{0}}^{T} \gamma(t) e^{\int_{t_{0}}^{t} C_{R}\left[\left(1+\left|f\left(x_{n}(s), u_{n}(s)\right)\right|+L\left(x_{n}(s), u_{n}(s)\right)^{1 / \alpha}\right] d s\right.} d t \\
& \quad \leq \int_{t_{0}}^{T} C_{R}\left|x_{n}\left(t_{0}\right)-y_{n}^{h}\left(t_{0}\right)\right|\left[1+\left|f\left(x_{n}(t), u_{n}(t)\right)\right|+L\left(x_{n}(t), u_{n}(t)\right)^{1 / \alpha}\right] e^{C_{R} C} d t \\
& \quad \leq C_{1}\left|x_{n}\left(t_{0}\right)-y_{n}^{h}\left(t_{0}\right)\right| \leq R .
\end{aligned}
$$

Applying Filippov's Theorem (see, e.g., 12] ) we deduce that there exists a solution $z_{n}^{h}$ of A.10 defined on $\left[t_{0}, T\right]$ such that, for every $t \in\left[t_{0}, T\right]$ and some $C$ independent from $n$,

$$
\left|z_{n}^{h}(t)-z(t)\right| \leq C\left|x_{n}\left(t_{0}\right)-y_{n}^{h}\left(t_{0}\right)\right|
$$

Then, the function

$$
x_{n}^{h}(t)= \begin{cases}x_{n}(t) & \text { for } t \in\left[0, t_{0}-h\right], \\ y_{n}^{h}(t) & \text { for } t \in\left[t_{0}-h, t_{0}\right], \\ z_{n}^{h}(t) & \text { for } t \in\left[t_{0}, T\right]\end{cases}
$$

satisfies (A.8) on $I$. Moreover, there exists $R>0$ such that $x_{n}(t), x_{n}^{h}(t) \in B(0, R)$, for every $t \in I$ and every $n \in \mathbb{N}$. Now, observe that, for a.e. $t \in I$,

$$
\begin{aligned}
\mid x_{n}^{\prime}(t)- & \left(x_{n}^{h}\right)^{\prime}(t) \mid \\
\leq & \left|f\left(x_{n}(t), u_{n}(t)\right)-f\left(x_{n}(t), u_{n}^{h}(t)\right)\right|+\left|f\left(x_{n}(t), u_{n}^{h}(t)\right)-f\left(x_{n}^{h}(t), u_{n}^{h}(t)\right)\right| \\
\leq & \left|f\left(x_{n}(t), u_{n}(t)\right)-f\left(x_{n}(t), u_{n}^{h}(t)\right)\right| \\
& \quad+C_{R}\left|x_{n}(t)-x_{n}^{h}(t)\right|\left[1+\left|f\left(x_{n}(t), u_{n}^{h}(t)\right)\right|+L\left(x_{n}(t), u_{n}^{h}(t)\right)^{1 / \alpha}\right],
\end{aligned}
$$

and, for some $C>0$,

$$
\int_{I}\left[1+\left|f\left(x_{n}(t), u_{n}^{h}(t)\right)\right|+L\left(x_{n}(t), u_{n}^{h}(t)\right)^{1 / \alpha}\right] d t \leq C .
$$

From Gronwall's Lemma it follows that, for some $C>0$,

$$
\begin{aligned}
\left\|x_{n}-x_{n}^{h}\right\|_{\infty} & \leq C \int_{0}^{T}\left|f\left(x_{n}(s), u_{n}(s)\right)-f\left(x_{n}(s), u_{n}^{h}(s)\right)\right| d s \\
& =C \int_{t_{0}-h}^{t_{0}}\left|f\left(x_{n}(s), u_{n}(s)\right)-f\left(x_{n}(s), \bar{u}(s)\right)\right| d s .
\end{aligned}
$$


In view of $(\mathrm{H}) \mathrm{i})$ we have that

$$
\begin{aligned}
\int_{0}^{T} L\left(x_{n}^{h}(t), u_{n}^{h}(t)\right) d t \leq & \int_{0}^{T} L\left(x_{n}(t), u_{n}^{h}(t)\right) d t \\
& +\int_{0}^{T} C_{R}\left|x_{n}(t)-x_{n}^{h}(t)\right|\left[1+L\left(x_{n}(t), u_{n}^{h}(t)\right)\right] d t \\
\leq & \int_{0}^{T} L\left(x_{n}(t), u_{n}^{h}(t)\right) d t+C_{R}\left\|x_{n}-x_{n}^{h}\right\|_{\infty}(T+C) .
\end{aligned}
$$

On the other hand, for a.e. $t \in I$,

$$
\begin{aligned}
& \left|f\left(x_{n}^{h}(t), u_{n}^{h}(t)\right)-f\left(x_{n}(t), u_{n}^{h}(t)\right)\right| \\
& \quad \leq C_{R}\left|x_{n}(t)-x_{n}^{h}(t)\right|\left[1+\left|f\left(x_{n}(t), u_{n}^{h}(t)\right)\right|+L\left(x_{n}(t), u_{n}^{h}(t)\right)^{1 / \alpha}\right] \\
& \quad \leq C_{R}\left\|x_{n}-x_{n}^{h}\right\|_{\infty}\left[1+\left|f\left(x_{n}(t), u_{n}^{h}(t)\right)\right|+L\left(x_{n}(t), u_{n}^{h}(t)\right)^{1 / \alpha}\right]
\end{aligned}
$$

and

$$
\begin{aligned}
& \left|f\left(x_{n}^{h}(t), u_{n}^{h}(t)\right)\right|+\left|f\left(x_{n}(t), u_{n}^{h}(t)\right)\right| \\
& \leq 2\left|f\left(x_{n}(t), u_{n}^{h}(t)\right)\right|+C_{R}\left|x_{n}(t)-x_{n}^{h}(t)\right|\left[1+\left|f\left(x_{n}(t), u_{n}^{h}(t)\right)\right|+L\left(x_{n}(t), u_{n}^{h}(t)\right)^{1 / \alpha}\right] \\
& \leq 2\left|f\left(x_{n}(t), u_{n}^{h}(t)\right)\right|+C_{R}\left\|x_{n}-x_{n}^{h}\right\|_{\infty}\left[1+\left|f\left(x_{n}(t), u_{n}^{h}(t)\right)\right|+L\left(x_{n}(t), u_{n}^{h}(t)\right)^{1 / \alpha}\right] .
\end{aligned}
$$

Moreover, from the boundedness of $\left\{f\left(x_{n}(\cdot), u_{n}(\cdot)\right)\right\}_{n \in \mathbb{N}}$ in $L^{\alpha}$ and from the definition of $u_{n}^{h}$, for $1 / \alpha+1 / \alpha^{\prime}=1$ and for some $C_{\alpha}, C_{\alpha^{\prime}}>0$,

$$
\left\|1+\left|f\left(x_{n}(\cdot), u_{n}^{h}(\cdot)\right)\right|+L\left(x_{n}(\cdot), u_{n}^{h}(\cdot)\right)^{1 / \alpha}\right\|_{L^{\alpha}} \leq C_{\alpha}
$$

and

$$
\left\|\left(\left|f\left(x_{n}^{h}(\cdot), u_{n}^{h}(\cdot)\right)\right|+\left|f\left(x_{n}(\cdot), u_{n}^{h}(\cdot)\right)\right|\right)^{\alpha-1}\right\|_{L^{\alpha^{\prime}}} \leq C_{\alpha^{\prime}} .
$$

Since

$$
a^{\alpha}-b^{\alpha}=\int_{b}^{a} \alpha t^{\alpha-1} d t \leq \alpha|a-b|(a+b)^{\alpha-1}, \quad \text { for every } a, b \geq 0,
$$

applying (A.12)- A.16) and Hölder's inequality, we obtain that

$$
\begin{aligned}
& \frac{\epsilon}{\alpha} \int_{0}^{T}\left|f\left(x_{n}^{h}(t), u_{n}^{h}(t)\right)\right|^{\alpha} d t \leq \frac{\epsilon}{\alpha} \int_{0}^{T}\left|f\left(x_{n}(t), u_{n}^{h}(t)\right)\right|^{\alpha} d t \\
& +\epsilon \int_{0}^{T}\left|f\left(x_{n}^{h}(t), u_{n}^{h}(t)\right)-f\left(x_{n}(t), u_{n}^{h}(t)\right)\right|\left(\left|f\left(x_{n}^{h}(t), u_{n}^{h}(t)\right)\right|+\left|f\left(x_{n}(t), u_{n}^{h}(t)\right)\right|\right)^{\alpha-1} d t \\
& \leq \frac{\epsilon}{\alpha} \int_{0}^{T}\left|f\left(x_{n}(t), u_{n}^{h}(t)\right)\right|^{\alpha} d t+\epsilon C_{R}\left\|x_{n}-x_{n}^{h}\right\|_{\infty} C_{\alpha} C_{\alpha^{\prime}} .
\end{aligned}
$$


Now, applying (A.11) it follows that, for some constants $C, C_{1}$,

$$
\begin{aligned}
\tilde{J}_{\epsilon}\left(u_{n}^{h}\right)= & \int_{0}^{T} L_{\epsilon}\left(x_{n}^{h}(t), u_{n}^{h}(t)\right) d t+\ell\left(x_{n}^{h}(T)\right) \\
\leq & \int_{0}^{T} L_{\epsilon}\left(x_{n}(t), u_{n}^{h}(t)\right) d t+\ell\left(x_{n}(T)\right)+C\left\|x_{n}-x_{n}^{h}\right\|_{\infty} \\
\leq & \tilde{J}_{\epsilon}\left(u_{n}\right)+\int_{t_{0}-h}^{t_{0}}\left[L_{\epsilon}\left(x_{n}(t), \bar{u}(t)\right)-L_{\epsilon}\left(x_{n}(t), u_{n}(t)\right)\right] d t \\
& +C_{1} \int_{t_{0}-h}^{t_{0}}\left|f\left(x_{n}(t), u_{n}(t)\right)-f\left(x_{n}(t), \bar{u}(t)\right)\right| d t .
\end{aligned}
$$

By (A.7) we have that $\tilde{J}_{\epsilon}\left(u_{n}\right)=J_{\epsilon}^{n}\left(u_{n}\right) \leq J_{\epsilon}^{n}\left(u_{n}^{h}\right) \leq \tilde{J}_{\epsilon}\left(u_{n}^{h}\right)+\sqrt{\delta_{n}} h$. Hence,

$$
\begin{aligned}
0 \leq & \int_{t_{0}-h}^{t_{0}}\left[L_{\epsilon}\left(x_{n}(t), \bar{u}(t)\right)-L_{\epsilon}\left(x_{n}(t), u_{n}(t)\right)\right] d t \\
& +C \int_{t_{0}-h}^{t_{0}}\left|f\left(x_{n}(t), u_{n}(t)\right)-f\left(x_{n}(t), \bar{u}(t)\right)\right| d t+\sqrt{\delta_{n}} h .
\end{aligned}
$$

Since $t_{0}$ is a Lebesgue point for

$$
L_{\epsilon}\left(x_{n}(\cdot), \bar{u}(\cdot)\right)-L_{\epsilon}\left(x_{n}(\cdot), u_{n}(\cdot)\right) \quad \text { and } \quad\left|f\left(x_{n}(\cdot), u_{n}(\cdot)\right)-f\left(x_{n}(\cdot), \bar{u}(\cdot)\right)\right|,
$$

dividing by $h$ and taking the limit $h \rightarrow 0$, we obtain that

$$
\begin{aligned}
0 \leq L_{\epsilon}\left(x_{n}\left(t_{0}\right), \bar{u}\left(t_{0}\right)\right) & -L_{\epsilon}\left(x_{n}\left(t_{0}\right), u_{n}\left(t_{0}\right)\right) \\
& +C\left|f\left(x_{n}\left(t_{0}\right), u_{n}\left(t_{0}\right)\right)-f\left(x_{n}\left(t_{0}\right), \bar{u}\left(t_{0}\right)\right)\right|+\sqrt{\delta_{n}} .
\end{aligned}
$$

Therefore,

$$
\begin{aligned}
L_{\epsilon}\left(x_{n}\left(t_{0}\right), u_{n}\left(t_{0}\right)\right) \leq L_{\epsilon}\left(x_{n}\left(t_{0}\right), \bar{u}\left(t_{0}\right)\right) & +C\left|f\left(x_{n}\left(t_{0}\right), u_{n}\left(t_{0}\right)\right)\right| \\
& +C\left|f\left(x_{n}\left(t_{0}\right), \bar{u}\left(t_{0}\right)\right)\right|+\sqrt{\delta_{n}} .
\end{aligned}
$$

By Lemma 2.5, $\left\{f\left(x_{n}(\cdot), u_{n}(\cdot)\right)\right\}_{n \in \mathbb{N}}$ is equi-integrable in $I$. So, using assumptions (H) ii) and iii) we deduce that $\left\{L_{\epsilon}\left(x_{n}(\cdot), u_{n}(\cdot)\right)\right\}_{n \in \mathbb{N}}$ is equi-integrable. Since $\left\{L_{\epsilon}\left(x_{n}(\cdot), u_{n}(\cdot)\right)\right\}_{n \in \mathbb{N}}$ is also bounded in $L^{1}$, invoking the Dunford-Pettis Theorem and taking again a subsequence, we may assume that $\left\{L_{\epsilon}\left(x_{n}(\cdot), u_{n}(\cdot)\right)\right\}_{n \in \mathbb{N}}$ converges weakly in $L^{1}$ to some $\xi \in L^{1}\left(I ; \mathbb{R}^{N}\right)$.

Step 4 . We have shown that there exists $x_{\epsilon} \in W^{1,1}\left(I ; \mathbb{R}^{N}\right)$ with $x_{\epsilon}(0) \in C_{0}$ and a function $\xi \in L^{1}\left(I ; \mathbb{R}^{N}\right)$ such that, when $n \rightarrow+\infty$,

$$
\begin{cases}x_{n} \rightarrow x_{\epsilon} & \text { uniformly, } \\ x_{n}^{\prime} \rightarrow x_{\epsilon}{ }^{\prime} & \text { weakly in } L^{1} \\ L_{\epsilon}\left(x_{n}(\cdot), u_{n}(\cdot)\right) \rightarrow \xi & \text { weakly in } L^{1}\end{cases}
$$

Working as in Step 3 in the proof of Theorem 3.1, we obtain that there exist two measurable functions $u_{\epsilon}, v_{\epsilon}$ such that, for a.e. $t \in I, u_{\epsilon}(t) \in U, v_{\epsilon}(t) \geq 0$ and

$$
\left\{\begin{array}{l}
x_{\epsilon}{ }^{\prime}(t)=f\left(x_{\epsilon}(t), u_{\epsilon}(t)\right) \\
\xi(t)=L_{\epsilon}\left(x_{\epsilon}(t), u_{\epsilon}(t)\right)+v_{\epsilon}(t) .
\end{array}\right.
$$

Since, by (A.17) and (A.18),

$$
\int_{0}^{T}\left[L_{\epsilon}\left(x_{\epsilon}(t), u_{\epsilon}(t)\right)+v_{\epsilon}(t)\right] d t+\ell\left(x_{\epsilon}(T)\right) \leq \lim _{n \rightarrow+\infty} J_{\epsilon}\left(x_{n}, u_{n}\right)=\beta_{\epsilon},
$$


where $\beta_{\epsilon}$ is the infimum of problem (2.5), (1.2), it follows that $v_{\epsilon}(t)=0$, for a.e. $t \in I$. So, $\left(x_{\epsilon}, u_{\epsilon}\right)$ is an optimal solution, and, from (A.1),

$$
J\left(x_{\epsilon}, u_{\epsilon}\right) \leq J_{\epsilon}\left(x_{\epsilon}, u_{\epsilon}\right)<J\left(x_{1}, u_{1}\right),
$$

proving a).

Let $\left(x_{\epsilon}, u_{\epsilon}\right)$ be an optimal solution of problem (2.5), (1.2), and suppose that:

(h) there exist two Borel measurable functions $l, k: \mathbb{R}^{m} \rightarrow \mathbb{R}_{+}$, and $\delta>0$ such that $l\left(u_{\epsilon}(\cdot)\right), k\left(u_{\epsilon}(\cdot)\right) \in L^{1}(I ; \mathbb{R})$ and

$$
\left|L_{\epsilon}(x, u)-L_{\epsilon}(y, u)\right| \leq l(u)|x-y| \quad \text { and } \quad|f(x, u)-f(y, u)| \leq k(u)|x-y|,
$$

for every $u \in U$ and every $x, y \in B\left(X_{\epsilon}, \delta\right)$, where

$$
X_{\epsilon}=\left\{x_{\epsilon}(t): t \in I\right\} .
$$

Then, the Pontryagin Maximum Principle (see Vinter [12, p.203]) ensures that there exist $c_{\epsilon} \in \mathbb{R}$ and $p_{\epsilon} \in W^{1,1}\left(I ; \mathbb{R}^{N}\right)$ such that, for a.e. $t \in I$,

$$
\begin{aligned}
c_{\epsilon}= & \left\langle p_{\epsilon}(t), f\left(x_{\epsilon}(t), u_{\epsilon}(t)\right)\right\rangle-L_{\epsilon}\left(x_{\epsilon}(t), u_{\epsilon}(t)\right) \\
= & \max _{u \in U}\left\{\left\langle p_{\epsilon}(t), f\left(x_{\epsilon}(t), u\right)\right\rangle-L_{\epsilon}\left(x_{\epsilon}(t), u\right)\right\}, \\
-p_{\epsilon}^{\prime}(t) \in & \left(\partial_{x} f\right)^{*}\left(x_{\epsilon}(t), u_{\epsilon}(t)\right) p_{\epsilon}(t)-\partial_{x} L\left(x_{\epsilon}(t), u_{\epsilon}(t)\right) \\
& \quad-\epsilon\left|f\left(x_{\epsilon}(t), u_{\epsilon}(t)\right)\right|^{\alpha-1}\left(\partial_{x} f\right)^{*}\left(x_{\epsilon}(t), u_{\epsilon}(t)\right) \phi_{\varepsilon}(t),
\end{aligned}
$$

where $\phi_{\varepsilon}(t) \in B(0,1)$, and

$$
-p_{\epsilon}(T) \in \partial \ell\left(x_{\epsilon}(T)\right) .
$$

Let now $\delta=1$ and $R_{\epsilon}=\left\|x_{\epsilon}\right\|_{\infty}+1$. From (H) i) we have that, $\forall x \in B\left(0, R_{\epsilon}\right)$ and $\forall u \in U$,

$$
\begin{gathered}
|f(x, u)| \leq C_{R_{\epsilon}} R_{\epsilon}+|f(0, u)|\left(1+C_{R_{\epsilon}} R_{\epsilon}\right)+L(0, u)^{1 / \alpha} C_{R_{\epsilon}} R_{\epsilon}, \\
L(x, u) \leq C_{R_{\epsilon}} R_{\epsilon}+L(0, u)\left(1+C_{R_{\epsilon}} R_{\epsilon}\right),
\end{gathered}
$$

and, since $\alpha>1$,

$$
L(x, u)^{1 / \alpha} \leq\left(C_{R_{\epsilon}} R_{\epsilon}\right)^{1 / \alpha}+L(0, u)^{1 / \alpha}\left(1+C_{R_{\epsilon}} R_{\epsilon}\right)^{1 / \alpha} .
$$

Let $x, y \in B\left(X_{\epsilon}, 1\right) \subset B\left(0, R_{\epsilon}\right)$. From (A.23), (A.24), and (H) i), we obtain that $\forall u \in U$,

$$
|f(x, u)-f(y, u)| \leq C_{R_{\epsilon}}|x-y|\left[1+|f(x, u)|+L(x, u)^{1 / \alpha}\right] \leq k(u)|x-y|,
$$

where $k(u)=C_{f}\left[1+|f(0, u)|+L(0, u)^{1 / \alpha}\right]$, for a constant $C_{f}$ depending only on $R_{\epsilon}, C_{R_{\epsilon}}$. Moreover, from (A.16) and (A.23) it follows that

$$
\begin{aligned}
& \left.|| f(x, u)\right|^{\alpha}-|f(y, u)|^{\alpha}|\leq \alpha| f(x, u)-f(y, u) \mid(|f(x, u)|+|f(y, u)|)^{\alpha-1} \\
& \quad \leq \alpha k(u)|x-y|\left[2|f(0, u)|+2 C_{R_{\epsilon}} R_{\epsilon}\left(1+|f(0, u)|+L(0, u)^{1 / \alpha}\right)\right]^{\alpha-1} .
\end{aligned}
$$

We deduce that

$$
\begin{aligned}
\mid L_{\epsilon}(x, u) & -L_{\epsilon}(y, u)|\leq| L(x, u)-L(y, u)\left|+\frac{\epsilon}{\alpha}\right||f(x, u)|^{\alpha}-|f(y, u)|^{\alpha} \mid \\
& \leq C_{R_{\epsilon}}|x-y|(1+L(x, u))+\left.\frac{\epsilon}{\alpha}|| f(x, u)\right|^{\alpha}-|f(y, u)|^{\alpha}|\leq l(u)| x-y \mid,
\end{aligned}
$$


where $l(u)=C_{l}\left[1+L(0, u)+\left[1+|f(0, u)|+L(0, u)^{1 / \alpha}\right]^{\alpha}\right]$, for a constant $C_{l}$ depending only on $R_{\epsilon}, C_{R_{\epsilon}}$. We claim that, for this choice of $k$ and $l$, assumption (h) is satisfied. Indeed, since $\left|f\left(x_{\epsilon}(\cdot), u_{\epsilon}(\cdot)\right)\right|$ and $L\left(x_{\epsilon}(\cdot), u_{\epsilon}(\cdot)\right)^{1 / \alpha}$ belong to $L^{\alpha}(I ; \mathbb{R})$, an easy computation provides that $k\left(u_{\epsilon}(\cdot)\right)$ and $l\left(u_{\epsilon}(\cdot)\right)$ belong to $L^{1}(I ; \mathbb{R})$. So, the Pontryagin Maximum Principle holds and b) is proved.

From (A.19) and the assumptions of Lemma 2.6, for some $C>0$ and every $\epsilon \in\left(0, \epsilon_{0}\right)$,

$$
\left\|L\left(x_{\epsilon}(\cdot), u_{\epsilon}(\cdot)\right)\right\|_{L^{1}} \leq C \quad \text { and } \quad\left\|x_{\epsilon}\right\|_{\infty} \leq k .
$$

Using (H) i) and (A.21) we obtain, for a.e. $t \in I$,

$$
\begin{aligned}
\left|p_{\epsilon}^{\prime}(t)\right| & \leq C_{k}\left[1+\left|f\left(x_{\epsilon}(t), u_{\epsilon}(t)\right)\right|+L\left(x_{\epsilon}(t), u_{\epsilon}(t)\right)^{1 / \alpha}\right]\left|p_{\epsilon}(t)\right| \\
& +C_{k}\left[1+L\left(x_{\epsilon}(t), u_{\epsilon}(t)\right)\right] \\
& +\epsilon C_{k}\left[1+\left|f\left(x_{\epsilon}(t), u_{\epsilon}(t)\right)\right|+L\left(x_{\epsilon}(t), u_{\epsilon}(t)\right)^{1 / \alpha}\right]\left|f\left(x_{\epsilon}(t), u_{\epsilon}(t)\right)\right|^{\alpha-1} .
\end{aligned}
$$

Setting, for $t \in I, b_{\epsilon}(t):=1+\left|f\left(x_{\epsilon}(t), u_{\epsilon}(t)\right)\right|+L\left(x_{\epsilon}(t), u_{\epsilon}(t)\right)^{1 / \alpha}$, from (A.26) we obtain

$$
\left|p_{\epsilon}^{\prime}(t)\right| \leq C_{k} b_{\epsilon}(t)\left|p_{\epsilon}(t)\right|+\left(1+\epsilon_{0}\right) C_{k} b_{\epsilon}^{\alpha}(t) .
$$

Assume that, for some $C>0$ and every $\epsilon \in\left(0, \epsilon_{0}\right)$,

$$
\left\|f\left(x_{\epsilon}(\cdot), u_{\epsilon}(\cdot)\right)\right\|_{L^{\alpha}}<C .
$$

(A.25) and A.27) imply that $\exists b_{1}, b_{\alpha}>0$ independent of $\epsilon$ such that $\left\|b_{\epsilon}\right\|_{L^{1}} \leq$ $b_{1},\left\|b_{\epsilon}\right\|_{L^{\alpha}} \leq b_{\alpha}$. Moreover, by the $C_{k^{-}}$-Lipschitzianity of $\ell(\cdot)$ and by (A.22), we obtain that $\left|p_{\epsilon}(T)\right| \leq C_{k}$ so that, applying Gronwall's Lemma, we deduce, for every $t \in I$,

$$
\begin{aligned}
\left|p_{\epsilon}(t)\right| & \leq e^{\int_{t}^{T} C_{k} b_{\epsilon}(s) d s}\left[p_{\epsilon}(T)+\int_{t}^{T} e^{-\int_{s}^{T} C_{k} b_{\epsilon}(\tau) d \tau}\left(1+\epsilon_{0}\right) C_{k} b_{\epsilon}^{\alpha}(s) d s\right] \\
& \leq e^{C_{k} b_{1}}\left[C_{k}+\left(1+\epsilon_{0}\right) C_{k} b_{\alpha}^{\alpha}\right] .
\end{aligned}
$$

Furthermore, if for a sequence $\epsilon_{n} \rightarrow 0^{+}$,

$$
\sup _{n \in \mathbb{N}}\left[\left\|L\left(x_{\epsilon_{n}}(\cdot), u_{\epsilon_{n}}(\cdot)\right)\right\|_{\infty}+\left\|f\left(x_{\epsilon_{n}}(\cdot), u_{\epsilon_{n}}(\cdot)\right)\right\|_{\infty}\right]<+\infty,
$$

then, from (A.26) and (A.28), also $\left\{p_{\epsilon_{n}}^{\prime}\right\}_{n \in \mathbb{N}}$ is bounded in $L^{\infty}$. This completes the proof.

Proof of Lemma 2.7. Since $\left(\mathrm{H}^{\prime}\right)$ yields $(\mathrm{H})$, all the arguments of the proof of Lemma 2.6 are still valid under assumptions $\left(\mathrm{H}^{\prime}\right)$. From (A.19) and the assumptions of Lemma 2.6

$$
\left\|L\left(x_{\epsilon}(\cdot), u_{\epsilon}(\cdot)\right)\right\|_{L^{1}} \leq C, \quad \epsilon\left\|f\left(x_{\epsilon}(\cdot), u_{\epsilon}(\cdot)\right)\right\|_{L^{1}}^{\alpha} \leq C \quad \text { and } \quad\left\|x_{\epsilon}\right\|_{\infty} \leq k,
$$
for some $C>0$ and every $\epsilon \in\left(0, \epsilon_{0}\right)$. $\left(\mathrm{H}^{\prime}\right)$ and (A.21) imply that, for a.e. $t \in I$,

$$
\begin{aligned}
\left|p_{\epsilon}^{\prime}(t)\right| \leq & C_{k}\left[1+L\left(x_{\epsilon}(t), u_{\epsilon}(t)\right)^{1 / \alpha}\right]\left|p_{\epsilon}(t)\right|+C_{k}\left[1+L\left(x_{\epsilon}(t), u_{\epsilon}(t)\right)\right] \\
& +C_{k}\left[1+L\left(x_{\epsilon}(t), u_{\epsilon}(t)\right)^{1 / \alpha}\right] \epsilon\left|f\left(x_{\epsilon}(t), u_{\epsilon}(t)\right)\right|^{\alpha-1} \\
\leq & C_{k} d_{\epsilon}(t)\left|p_{\epsilon}(t)\right|+2 C_{k} d_{\epsilon}^{\alpha}(t),
\end{aligned}
$$


where, for $t \in I, d_{\epsilon}(t):=1+\epsilon^{1 / \alpha}\left|f\left(x_{\epsilon}(t), u_{\epsilon}(t)\right)\right|+L\left(x_{\epsilon}(t), u_{\epsilon}(t)\right)^{1 / \alpha}$. A.29) implies that there exist $d_{1}, d_{\alpha}>0$ independent of $\epsilon$ such that $\left\|d_{\epsilon}\right\|_{L^{1}} \leq d_{1},\left\|d_{\epsilon}\right\|_{L^{\alpha}} \leq d_{\alpha}$. By the $C_{k}$-Lipschitzianity of $\ell$ on $B(0, k)$ and by (A.22), we have that $\left|p_{\epsilon}(T)\right| \leq C_{k}$. So, applying Gronwall's Lemma, we deduce

$$
\begin{aligned}
\left|p_{\epsilon}(t)\right| & \leq e^{\int_{t}^{T} C_{k} d_{\epsilon}(s) d s}\left[p_{\epsilon}(T)+\int_{t}^{T} e^{-\int_{s}^{T} C_{k} d_{\epsilon}(\tau) d \tau} 2 C_{k} d_{\epsilon}^{\alpha}(s) d s\right] \\
& \leq e^{C_{k} d_{1}}\left[C_{k}+2 C_{k} d_{\alpha}^{\alpha}\right],
\end{aligned}
$$

for every $t \in I$. This completes the proof.

\section{REFERENCES}

1. J.-P. Aubin, H. Frankowska, Set-Valued Analysis, Birkhäuser, Boston, 1990. MR1048347 (91d:49001)

2. A. Cellina, The classical problem of the calculus of variations in the autonomous case: Relaxation and Lipschitzianity of solutions, Trans. Amer. Math. Soc., 356 (2004), 1, 415-426. MR 2020039 (2004k:49087)

3. A. Cellina, A. Ferriero, Existence of Lipschitzian solutions to the classical problem of the calculus of variations in the autonomous case, Ann. Inst. H. Poincaré Anal. Non Linéaire, 20 (2003), 6, 911-919. MR2008683 (2004f:49069)

4. L. Cesari, Optimization, Theory and Applications, Springer, Berlin, 1983. MR688142 (85c:49001)

5. F. H. Clarke, An indirect method in the Calculus of Variations, Trans. Amer. Math. Soc., 336 (1993), 2, 655-673. MR1118823 (93f:49002)

6. F. H. Clarke, Optimization and Nonsmooth Analysis, Wiley-Interscience, New York, 1983. MR709590 (85m:49002)

7. F. H. Clarke, R. B. Vinter, Regularity properties of optimal controls, SIAM J. Control Optim., 28 (1990), 4, 980-997. MR1051634 (91b:49033)

8. N. Dunford and J. Schwartz, Linear operators, Part I, General Theory, Interscience, New York, 1967. MR1009162 (90g:47001a)

9. H. Frankowska, E. M. Marchini, Lipschitzianity of optimal trajectories for the Bolza optimal control problem, Calc. Var. Partial Differential Equations, 27 (2006), 4, 467-492. MR2263674 (2007f:49021)

10. A. V. Sarychev, D. F. M. Torres, Lipschitzian regularity of the minimizers for optimal control problems with control-affine dynamics, Appl. Math. Optim., 41 (2000), 2, 237-254. MR $1731420(2000 \mathrm{~m}: 49048)$

11. D. F. M. Torres, Lipschitzian regularity of the minimizing trajectories for nonlinear optimal control problems, Math. Control Signals Systems, 16 (2003), 2-3, 158-174. MR2006825 (2004i:49047)

12. R. B. Vinter, Optimal control, Birkhäuser, Boston, Basel, Berlin, 2000. MR 1756410 (2001c:49001)

Dipartimento di Matematica, Università di Roma "Tor Vergata", Via della Ricerca Scientifica 1, 00133 Roma, Italy

E-mail address: cannarsa@axp.mat.uniroma2.it

Combinatoire et Optimisation, Université Pierre et Marie Curie (Paris 6) Case 189, 4 Place Jussieu, 75252 Paris Cedex 05, France

E-mail address: frankowska@math.jussieu.fr

Dipartimento di Matematica, Politecnico di Milano, Piazza leonardo Da Vinci 32, 20133 Milano, Italy

E-mail address: elsa.marchini@polimi.it 